\title{
Planck intermediate results. XVIII. The millimetre and sub-millimetre emission from planetary nebulae
}

Planck Collaboration: M. Arnaud ${ }^{64}$, F. Atrio-Barandela ${ }^{16}$, J. Aumont ${ }^{52}$, C. Baccigalupi ${ }^{74}$, A. J. Banday ${ }^{80,9}$, R. B. Barreiro ${ }^{58}$, E. Battaner ${ }^{81,82}$, K. Benabed ${ }^{53,78}$, A. Benoit-Lévy ${ }^{21,53,78}$, J.-P. Bernard ${ }^{80,9}$, M. Bersanelli ${ }^{30,46}$, P. Bielewicz ${ }^{80,9,74}$, A. Bonaldi ${ }^{60}$, J. R. Bond ${ }^{8}$, J. Borrill ${ }^{12,76}$, F. R. Bouchet ${ }^{53,78}$, C. S. Buemi4 ${ }^{40}$, C. Burigana ${ }^{45,28,47}$ J.-F. Cardoso ${ }^{65,1,53}$, S. Casassus ${ }^{79}$, A. Catalano ${ }^{66,63}$, L. Cerrigone ${ }^{11}$, A. Chamballu ${ }^{64,13,52}$, H. C. Chiang ${ }^{24,7}$, S. Colombi ${ }^{53,78}$, L. P. L. Colombo ${ }^{20,59}$, F. Couchot $^{62}$, B. P. Crill ${ }^{59,72}$, A. Curto ${ }^{6,58}$, F. Cuttaia ${ }^{45}$, R. D. Davies ${ }^{60}$, R. J. Davis ${ }^{60}$, P. de Bernardis ${ }^{29}$, A. de Rosa ${ }^{45}$, G. de Zotti ${ }^{41,74}$, J. Delabrouille ${ }^{1}$, C. Dickinson ${ }^{60}$, J. M. Diego ${ }^{58}$, S. Donzelli ${ }^{46}$, O. Doré ${ }^{59,10}$, X. Dupac ${ }^{35}$, T. A. Enßlin ${ }^{69}$, H. K. Eriksen ${ }^{56}$, F. Finelli ${ }^{45,47}$, M. Frailis ${ }^{43}$, E. Franceschi ${ }^{45}$, S. Galeotta ${ }^{43}$, K. Ganga ${ }^{1}$, M. Giard ${ }^{80,9}$, J. González-Nuevo ${ }^{58,74}$, K. M. Górski ${ }^{59,83}$, A. Gregorio ${ }^{31,43,49}$, A. Gruppuso ${ }^{45}$, F. K. Hansen ${ }^{56}$, D. L. Harrison ${ }^{55,61}$, S. R. Hildebrandt ${ }^{59}$, E. Hivon ${ }^{53,78}$, W. A. Holmes ${ }^{59}$, J. L. Hora ${ }^{38}$, A. Hornstrup ${ }^{14}$, W. Hovest ${ }^{69}$, K. M. Huffenberger ${ }^{22}$, T. R. Jaffe ${ }^{80,9}$, W. C. Jones ${ }^{24}$, M. Juvela ${ }^{23}$, E. Keihänen ${ }^{23}$, R. Keskitalo ${ }^{12}$,

T. S. Kisner ${ }^{68}$, J. Knoche ${ }^{69}$, M. Kunz ${ }^{15,52,3}$, H. Kurki-Suonio ${ }^{23,39}$, A. Lähteenmäki ${ }^{2,39}$, J.-M. Lamarre ${ }^{63}$, A. Lasenby ${ }^{6,61}$, C. R. Lawrence ${ }^{59}$, R. Leonardi ${ }^{35}$, P. Leto $^{40}$, P. B. Lilje ${ }^{56}$, M. Linden-Vørnle ${ }^{14}$, M. López-Caniego ${ }^{58}$, J. F. Macías-Pérez ${ }^{66}$, B. Maffei ${ }^{60}$, D. Maino ${ }^{30,46}$, N. Mandolesi ${ }^{45,5,28}$, P. G. Martin ${ }^{8}$, S. Masi ${ }^{29}$, M. Massardi ${ }^{44}$,

S. Matarrese ${ }^{27}$, P. Mazzotta ${ }^{32}$, L. Mendes ${ }^{35}$, A. Mennella ${ }^{30,46}$, M. Migliaccio ${ }^{55,61}$, M.-A. Miville-Deschênes ${ }^{52,8}$, A. Moneti ${ }^{53}$, L. Montier ${ }^{80,9}$, G. Morgante ${ }^{45}$, D. Mortlock ${ }^{50}$, D. Munshi ${ }^{75}$, J. A. Murphy ${ }^{70}$, P. Naselsky ${ }^{71,33}$, F. Nati ${ }^{29}$, P. Natoli ${ }^{28,4,45}$, F. Noviello ${ }^{60}$, D. Novikov ${ }^{50}$, I. Novikov ${ }^{71}$, L. Pagano ${ }^{29,48}$, F. Pajot ${ }^{52}$, R. Paladini ${ }^{51}$, D. Paoletti ${ }^{45,47}$,

M. Peel ${ }^{60}$, O. Perdereau ${ }^{62}$, F. Perrotta ${ }^{74}$, F. Piacentini ${ }^{29}$, M. Piat ${ }^{1}$, D. Pietrobon ${ }^{59}$, S. Plaszczynski ${ }^{62}$,

E. Pointecouteau ${ }^{80,9}$, G. Polenta ${ }^{4,42}$, L. Popa ${ }^{54}$, G. W. Pratt ${ }^{64}$, P. Procopio ${ }^{45}$, S. Prunet ${ }^{53,78}$, J.-L. Puget ${ }^{52}$,

J. P. Rachen ${ }^{18,69}$, M. Reinecke ${ }^{69}$, M. Remazeilles ${ }^{60,52,1}$, S. Ricciardi ${ }^{45}$, T. Riller ${ }^{69}$, I. Ristorcelli ${ }^{80,9}$, G. Rocha ${ }^{59,10}$,

C. Rosset $^{1}$, G. Roudier ${ }^{1,63,59}$, J. A. Rubiño-Martín ${ }^{57,34}$, B. Rusholme ${ }^{51}$, M. Sandri ${ }^{45}$, G. Savinii ${ }^{73}$, D. Scott ${ }^{19}$,

L. D. Spencer ${ }^{75}$, V. Stolyarov ${ }^{6,61,77}$, D. Sutton ${ }^{55,61}$, A.-S. Suur-Uski ${ }^{23,39}$, J.-F. Sygnet ${ }^{53}$, J. A. Tauber ${ }^{36}$, L. Terenzi $^{37,45}$,

L. Toffolatti ${ }^{17,58,45}$, M. Tomasi ${ }^{30,46}$, C. Trigilio ${ }^{40}$, M. Tristram ${ }^{62}$, T. Trombetti ${ }^{45}$, M. Tucci $^{15,62}$, G. Umana $^{40, \star}$, J. Valiviita ${ }^{23,39}$, B. Van Tent ${ }^{67}$, P. Vielva ${ }^{58}$, F. Villa ${ }^{45}$, L. A. Wade ${ }^{59}$, B. D. Wandelt ${ }^{53,78,26}$, A. Zacchei ${ }^{43}$, A. Zijlstra ${ }^{60}$, and A. Zonca ${ }^{25}$

(Affiliations can be found after the references)

Received 18 March 2014 / Accepted 1 October 2014

ABSTRACT

Late stages of stellar evolution are characterized by copious mass-loss events whose signature is the formation of circumstellar envelopes (CSE). Planck multi-frequency measurements have provided relevant information on a sample of Galactic planetary nebulae (PNe) in the important and relatively unexplored observational band between 30 and $857 \mathrm{GHz}$. Planck enables the assembly of comprehensive PNe spectral energy distributions (SEDs) from radio to far-IR frequencies. Modelling the derived SEDs provides us with information on physical properties of CSEs and the mass content of both main components: ionized gas, traced by the free-free emission at $\mathrm{cm}-\mathrm{mm}$ waves; and thermal dust, traced by the millimetre and far-IR emission. In particular, the amount of ionized gas and dust has been derived here. Such quantities have also been estimated for the very young PN CRL 618, where the strong variability observed in its radio and millimetre emission has previously prevented constructing its SED. A morphological study of the Helix Nebula was also performed. Planck maps reveal, for the first time, the spatial distribution of the dust inside the envelope, allowing us to identify different components, the most interesting of which is a very extended component (up to $1 \mathrm{pc}$ ) that may be related to a region where the slow expanding envelope is interacting with the surrounding interstellar medium.

Key words. planetary nebulae: general - radio continuum: ISM - submillimeter: ISM

\section{Introduction}

The final phases of low-to-intermediate mass stars are characterized by periods of high mass loss that lead to the formation of dense circumstellar envelopes (CSEs), where physical conditions are ideal for dust to condense (during the asymptotic giant

^ Corresponding author: G. Umana, e-mail: grazia.umana@oact.inaf.it branch, or AGB phase). Such envelopes can be very massive and, in some cases, the central object can be completely optically obscured. Eventually the mass loss stops and the central star becomes visible as the dusty shell disperses (the proto-planetary nebula, or PPN phase). During the subsequent evolutionary phases, the central star moves towards higher temperatures, and once the stellar temperature is high enough to ionize the surrounding medium, the object becomes a planetary nebula (PN; Kwok 2008). 
PNe are usually surrounded by a dusty envelope that is a remnant of the previous evolutionary phases and is partly ionized by the UV radiation from the central star. The material surrounding the central object consequently has quite a complex distribution, consisting of concentric shells. In these shells, the level of ionization decreases with the distance to the central star: highly ionized species are close to the star, while the outer part of the nebula is characterized by molecules and dust. This characteristic circumstellar environment implies the presence of two important components in the spectral energy distribution (SED) of a typical PN, whose major contributions fall in the spectral range from the far-IR to the radio region. The ionized fraction of the CSE can be traced by its free-free emission, which makes PNe bright Galactic radio sources, with some of them reaching flux densities up to a few Jy. Dust thermally re-radiates the absorbed stellar light, showing a clear signature in the far-IR spectrum, i.e., an IR excess in the colour-colour diagram created with data from the Infrared Astronomical Satellite (IRAS). Such a contribution is typically of the order of $40 \%$ of the total flux from a PN (Zhang \& Kwok 1991) and is larger in young PNe, since in more evolved PNe the circumstellar material has already dispersed.

$\mathrm{PNe}$ and their progenitors are considered to be among the major sources of recycled material into the ISM and for this reason, they are regarded as key objects for studying the chemical evolution of the Galaxy. Before being released into the ISM, significant processing of the material contained in the PN envelopes is expected. Gas and dust are exposed to a very harsh environment: shocks will occur when the fast outflows developing at the beginning of the PN phase overtake the slow expanding AGB envelope, and the UV radiation field radiated by the central star could be very intense, with consequences on the ionization/recombination equilibrium of the gas and on the dusty/molecular content of the envelope (Hora et al. 2009). It is therefore very important to establish not only the quantity of this processed material that is returned to the ISM after the nebula disperses, but also its general properties and dominant chemistry.

The aim of this work is to derive the physical characteristics of a sample of Galactic PNe, thereby taking advantage of the unique frequency coverage provided by the Planck ${ }^{1}$ measurements, which trace both the ionized and the dust components. We model the SEDs with particular attention to the continuum from the mid-IR to the radio. Results from such modelling will be used to derive important parameters of PN envelopes: the total mass, the ionized fraction, and the properties of the dust component. In some cases, hints of the extended morphology of PN envelopes can be derived from a direct inspection of the Planck maps.

As a starting point, we have compiled a master catalogue of Galactic PNe for which pre-existing $30 \mathrm{GHz}$ and/or $43 \mathrm{GHz}$ measurements are available. Our catalogue, based on single-dish measurements from the Torun $(30 \mathrm{GHz}$, Pazderska et al. 2009) and Noto (43 GHz, Umana et al. 2008a) surveys, consists of 119 $\mathrm{PNe}$ and covers a large parameter space in terms of location with respect to the Galactic plane, distance, and evolutionary stage. Typical numbers for the surveys are a full width at half maximum (FWHM) for the beam of $72^{\prime \prime}$ and an rms noise of $5 \mathrm{mJy}$ for the One Centimetre Receiver Array-prototype (OCRA-p)

\footnotetext{
1 Planck is a project of the European Space Agency - ESA - with instruments provided by two scientific Consortia funded by ESA member states (in particular the lead countries: France and Italy) with contributions from NASA (USA), and telescope reflectors provided in a collaboration between ESA and a scientific Consortium led and funded by Denmark.
}

observations, and a FWHM for the beam of $52^{\prime \prime}$ and rms noise of $70 \mathrm{mJy}$ for the Noto telescope survey.

This paper is organized as follows. Observations, consisting of Planck and ancillary data, are presented in Sect. 2, while the methods of extracting fluxes and results are described in Sect. 3. The adopted SED modelling and derived physical properties of the detected targets are illustrated in Sect. 4, while Sects. 5 and 6 focus on two targets, namely CLR 618 and NGC 7293 (the Helix Nebula, Helix hereafter), whose characterization appear to be particularly interesting. Section 7 concludes.

\section{Observations}

\subsection{Planck data}

Planck (Tauber et al. 2010; Planck Collaboration I 2011) is the third-generation space mission to measure the anisotropy of the cosmic microwave background (CMB). It observes the sky in nine frequency bands covering $30-857 \mathrm{GHz}$ with high sensitivity and angular resolution from $31^{\prime}$ to $5^{\prime}$. The Low Frequency Instrument (LFI; Mandolesi et al. 2010; Bersanelli et al. 2010; Mennella et al. 2011) covers the 30, 44, and $70 \mathrm{GHz}$ bands with amplifiers cooled to $20 \mathrm{~K}$. The High Frequency Instrument (HFI; Lamarre et al. 2010; Planck HFI Core Team 2011a) covers the $100,143,217,353,545$, and $857 \mathrm{GHz}$ bands with bolometers cooled to $0.1 \mathrm{~K}$. Polarization is measured in all but the highest two bands (Leahy et al. 2010; Rosset et al. 2010). A combination of radiative cooling and three mechanical coolers produces the temperatures needed for the detectors and optics (Planck Collaboration II 2011). Two data processing centres (DPCs) check and calibrate the data and make maps of the sky (Planck HFI Core Team 2011b; Zacchei et al. 2011). The sensitivity, angular resolution, and frequency coverage of Planck make it a powerful instrument for Galactic and extragalactic astrophysics as well as cosmology. Early astrophysics results are given in Planck Collaboration VIII (2011), Planck Collaboration IX (2011), Planck Collaboration X (2011), Planck Collaboration XI (2011), Planck Collaboration XII (2011), Planck Collaboration XIII (2011), Planck Collaboration XIV (2011), Planck Collaboration XV (2011), Planck Collaboration XVI (2011), Planck Collaboration XVII (2011), Planck Collaboration XVIII (2011), Planck Collaboration XIX (2011), Planck Collaboration XX (2011), Planck Collaboration XXI (2011), Planck Collaboration XXII (2011), Planck Collaboration XXIII (2011), Planck Collaboration XXIV (2011), Planck Collaboration XXV (2011), and Planck Collaboration XXVI (2011), based on data taken between 13 August 2009 and 7 June 2010. Intermediate astrophysics results are now being presented in a series of papers based on data taken between 13 August 2009 and 27 November 2010.

For this work we use the Planck Catalogue of Compact Sources (PCCS; Planck Collaboration XXVIII 2014) and the Planck maps from the 2013 distribution of released products (Planck Collaboration I 2014). Both products are based on data acquired during the "nominal" operations period from 13 August 2009 to 27 November 2010, and are available from the Planck Legacy Archive ${ }^{2}$. For the Planck maps we use here, the CMB thermodynamic units were converted to Rayleigh-Jeans brightness temperature units using the standard conversion factors described by Planck Collaboration I (2014). Some contamination due to $\mathrm{CO}$ emission is expected in the 100, 217, and, at a lower level, $353 \mathrm{GHz}$ Planck channels (Planck HFI Core Team 2011b), although this was not taken into account in the

\footnotetext{
http://WwW.sciops.esa.int/index.php? project=planck\&page=Planck_Legacy_Archive
} 
Planck Collaboration: The millimetre emission from planetary nebulae

Table 1. Non-colour-corrected flux densities (Jy) from Planck Catalogue of Compact Sources (PCCS; Planck Collaboration XXVIII 2014).

\begin{tabular}{|c|c|c|c|c|c|c|c|c|c|c|c|}
\hline \multirow[t]{2}{*}{ Source } & \multicolumn{3}{|l|}{ Coordinates } & \multicolumn{5}{|c|}{ Frequency (GHz) } & \multicolumn{3}{|r|}{ Ancillary data } \\
\hline & Galactic & 28.4 & 44.1 & 70.4 & 100 & 143 & 217 & 353 & 545 & 857 & Refs. \\
\hline NGC 6369 & $002.432+05.847$ & $1.9 \pm 0.2$ & $1.7 \pm 0.3$ & $1.27 \pm 0.15$ & $1.5 \pm 0.1$ & $1.27 \pm 0.061$ & $1.35 \pm 0.09$ & $2.4 \pm 0.3$ & $7.0 \pm 0.9$ & $\cdots$ & $\begin{array}{l}3,8,12,15,16,17,19,21,22,29,30,34,40,45,47 \\
50,54\end{array}$ \\
\hline NGC 6572 & $034.623+11.848$ & $1.1 \pm 0.1$ & $\cdots$ & $1.2 \pm 0.2$ & $0.96 \pm 0.06$ & $0.92 \pm 0.040$ & $0.81 \pm 0.04$ & $0.81 \pm 0.09$ & $\cdots$ & $\cdots$ & $\begin{array}{l}1,2,3,4,5,6,7,8,9,10,13,16,17,18,19,21,22,23 \\
26,29,30,32,33,34,35,38,39,48,50,54,55,56\end{array}$ \\
\hline NGC $7293^{\mathrm{a}}$ & $036.161-57.118$ & $0.8 \pm 0.1$ & $1.0 \pm 0.2$ & $0.88 \pm 0.15$ & $1.6 \pm 0.5$ & $1.6 \pm 0.5 \quad 3$ & $3.4 \pm 0.6$ & $10 \pm 1$ & $26 \pm 3$ & $73 \pm 9$ & $2,3,6,7,16,17,18,19,23,29,52$ \\
\hline NGC 7009 & $037.762-34.571$ & $0.6 \pm 0.1$ & $\cdots$ & $0.8 \pm 0.2$ & $0.54 \pm 0.06$ & $0.40 \pm 0.040$ & $0.42 \pm 0.04$ & $\cdots$ & $\cdots$ & $\cdots$ & $\begin{array}{l}2,3,6,8,9,16,17,21,26,29,30,41,45,47,50,51 \\
54,55,57,59\end{array}$ \\
\hline NGC 6853 & $060.836-03.696$ & $\cdots$ & $\cdots$ & $0.97 \pm 0.15$ & $0.87 \pm 0.07$ & $0.65 \pm 0.05$ & $\cdots$ & $\cdots$ & $\cdots$ & $\cdots$ & $\begin{array}{l}1,2,3,4,5,6,7,8,9,10,13,16,17,18,20,23,38 \\
50\end{array}$ \\
\hline NGC 6720 & $063.170+13.978$ & $\ldots$ & $\cdots$ & $\ldots$ & .. & $0.31 \pm 0.040$ & $0.33 \pm 0.03$ & $0.64 \pm 0.06$ & $1.6 \pm 0.1$ & $4.7 \pm 0.2$ & $\begin{array}{l}1,2,5,6,7,8,9,10,14,16,18,20,21,32,33,38,39 \\
48,50,55,60\end{array}$ \\
\hline NGC 6826 & $083.568+12.792$ & $0.35 \pm 0.08$ & $\cdots$ & $\cdots$ & $0.32 \pm 0.04$ & $0.25 \pm 0.030$ & $0.26 \pm 0.02$ & $\cdots$ & $\cdots$ & $\cdots$ & $6,8,10,12,16,17,21,33,38,39,48,50,55,60$ \\
\hline NGC 7027 & $084.930-03.496$ & $5.0 \pm 0.9$ & $4.7 \pm 0.6$ & $4.9 \pm 0.2$ & $4.4 \pm 0.1$ & $4.25 \pm 0.064$ & $4.3 \pm 0.1$ & $5.4 \pm 0.3$ & $6.3 \pm 0.7$ & $\cdots$ & $13,24,25,27,28,31,36,42,43,44,46,56$ \\
\hline NGC 6543 & $096.468+29.954$ & $0.74 \pm 0.08$ & $0.9 \pm 0.15$ & $0.67 \pm 0.09$ & $0.63 \pm 0.04$ & $0.52 \pm 0.020$ & $0.54 \pm 0.02$ & $0.59 \pm 0.04$ & $0.84 \pm 0.07$ & $2.6 \pm 0.2$ & $\begin{array}{l}2,4,6,7,8,16,17,21,22,27,38,39,47,48,49,50 \\
53,55,60\end{array}$ \\
\hline NGC 40 & $120.016+09.868$ & $\cdots$ & $\ldots$ & $0.78 \pm 0.15$ & $0.45 \pm 0.06$ & $0.36 \pm 0.040$ & $0.30 \pm 0.04$ & $\cdots$ & $\cdots$ & $\ldots$ & $6,10,12,16,17,38,39,48,50,60$ \\
\hline CRL 618 & $166.446-06.527$ & $0.7 \pm 0.2$ & $1.4 \pm 0.2$ & $1.9 \pm 0.2$ & $2.40 \pm 0.08$ & $2.67 \pm 0.053$ & $3.00 \pm 0.07$ & $4.9 \pm 0.2$ & $10.4 \pm 0.3$ & $24.3 \pm 0.8$ & $3^{32,56}$ \\
\hline IC 418 & $215.212-24.283$ & $1.6 \pm 0.1$ & $1.5 \pm 0.2$ & $1.4 \pm 0.2$ & $1.18 \pm 0.07$ & $1.02 \pm 0.040$ & $0.94 \pm 0.03$ & $0.84 \pm 0.08$ & $\ldots$ & $\ldots$ & $\begin{array}{l}2,3,4,6,7,8,9,10,11,13,15,16,17,19,21,22,30 \\
35,37,42,45,50,54,55,56,58,59\end{array}$ \\
\hline NGC 3242 & $261.051+32.050$ & $0.6 \pm 0.1$ & $\ldots$ & $\ldots$ & $0.51 \pm 0.06$ & $0.48 \pm 0.040$ & $0.37 \pm 0.03$ & $0.44 \pm 0.06$ & & $\cdots$ & $\begin{array}{l}2,3,4,6,8,9,10,11,12,13,16,17,21,22,29,35 \\
37,45,50,51,54,55,56,59\end{array}$ \\
\hline
\end{tabular}

Notes. ${ }^{(a)}$ Helix Nebula.

References. (1) Davies et al. (1965); (2) Menon \& Terzian (1965); (3) Slee \& Orchiston (1965); (4) Khromov (1966); (5) Terzian (1966); (6) Davies et al. (1967); (7) Hughes (1967); (8) Thompson et al. (1967); (9) Terzian (1968); (10) Kaftan-Kassim (1969); (11) Le Marne (1969); (12) Ribes (1969); (13) Terzian (1969); (14) Colla et al. (1970); (15) Rubin (1970); (16) Thomasson \& Davies (1970); (17) Higgs (1971); (18) Aller \& Milne (1972); (19) Higgs (1973); (20) Terzian \& Dickey (1973); (21) Sistla et al. (1974); (22) Terzian et al. (1974); (23) Milne \& Aller (1975); (24) Telesco \& Harper (1977); (25) Elias et al. (1978); (26) Cohen \& Barlow (1980); (27) Moseley (1980); (28) Ulich (1981); (29) Calabretta (1982); (30) Milne \& Aller (1982); (31) Gee et al. (1984); (32) Turner \& Terzian (1984); (33) Bennett et al. (1986); (34) Gathier et al. (1986); (35) Taylor et al. (1987); (36) Steppe et al. (1988); (37) Wright \& Otrupcek (1990); (38) Becker et al. (1991); (39) Gregory \& Condon (1991); (40) Large et al. (1991); (41) Wright et al. (1991); (42) Hoare et al. (1992); (43) Knapp et al. (1993); (44) Altenhoff et al. (1994); (45) Griffith et al. (1994); (46) Sandell (1994); (47) Douglas et al. (1996); (48) Gregory et al. (1996); (49) Rengelink et al. (1997); (50) Condon \& Kaplan (1998); (51) De Breuck et al. (2002); (52) Casassus et al. (2004); (53) Klaas et al. (2006); (54) Casassus et al. (2007); (55) Healey et al. (2007); (56) Di Francesco et al. (2008); (57) Vollmer et al. (2008); (58) Wright et al. (2009); (59) Murphy et al. (2010); (60) Vollmer et al. (2010).

following analysis. However, if some degree of CO contamination is present in our data, it is likely to amount only to a tiny fraction of the measured flux densities. In fact, as can be seen in the following analysis, in most cases the data points at each frequency are in very good agreement with the model and with the ancillary data, which only treat the continuum emission.

\subsection{Ancillary data}

To complete the SED for each of our selected targets, radio data from the literature (references reported in Table 1), as well as data from most infrared and radio all-sky surveys, such as the catalogues from the WMAP7-year data release (Gold et al. 2011), IRAS (Helou \& Walker 1988), MSX (Egan et al. 2003), Akari (Murakami et al. 2007), and WISE (Wright et al. 2010), have been collated.

To confirm the reliability of the IR and sub-mm flux densities, ancillary measurements in these wavelength ranges have been compared with the catalogue measurements for NGC 7027, a well-known radio/infrared flux-density calibrator. We found that the IRAS field around NGC 7027 is unavailable, but the MSX and Akari measurements match into a smooth SED with the SCUBA (Di Francesco et al. 2008) and the other ancillary targeted observations. The use of the WISE data needs instead some caution, because the sources studied in this paper can be too bright in the WISE bands, causing heavy saturation. Whenever WISE data are available, we use them after checking in each band the flags giving the fraction of pixels affected by saturation, a spurious detection or possible contamination.
For some sources of our sample, further sub-mm measurements are also available. If the aperture sizes are reported, as done for example by Hoare et al. (1992), we used the data only for those sources with angular sizes (given in Table 2) smaller than the aperture. Additional information about the $\mathrm{mm}$ and sub$\mathrm{mm}$ ancillary data is given in Table A.1.

The uncertainty estimates taken from the catalogues are of the order of $10 \%$ for IRAS, $5 \%$ for MSX, $10 \%$ for Akari and $3 \%$ for WISE, while for targeted observations we refer to the original papers. For one source, the Helix, we have also used IRIS $^{3}$ maps, which were retrieved via the on-line services of the IPAC Infrared Science Archive (IRSA). These are a new generation of IRAS images, which benefit from a better zodiacal light subtraction, as well as better calibration and destriping. The roughly $4^{\prime}$ resolution of the IRIS maps matches well with the high-frequency bands of Planck. Details on the whole processing and characterization of the IRIS data can be found in Miville-Deschênes \& Lagache (2005).

\section{Building the spectral energy distribution (SED)}

Compact sources reported in the PCCS were detected in each frequency channel map using a detection pipeline based on the Mexican Hat wavelet 2 (MHW2) algorithm (González-Nuevo et al. 2006; López-Caniego et al. 2006). Two independent implementations of the the MHW2 algorithm have been used by the LFI and HFI DPCs. More details on the PCCS can be found in

http://irsa.ipac.caltech.edu/data/IRIS/ 
Table 2. Source parameters for the free-free emission fit.

\begin{tabular}{|c|c|c|c|c|c|c|c|}
\hline \multirow[b]{2}{*}{ Source } & \multirow[b]{2}{*}{$\begin{array}{c}r_{\text {out }} \\
{[\operatorname{arcsec}]}\end{array}$} & \multirow[b]{2}{*}{$\begin{array}{c}\text { Distance } \\
{[\mathrm{pc}]}\end{array}$} & \multicolumn{3}{|c|}{ Free Parameters } & \multirow[b]{2}{*}{$\alpha$} & \multirow[b]{2}{*}{$\begin{array}{c}M_{\text {ion }} \\
{\left[M_{\odot}\right]}\end{array}$} \\
\hline & & & $\begin{array}{c}T \\
{\left[10^{4} \mathrm{~K}\right]}\end{array}$ & $r_{\text {int }} / r_{\text {out }}$ & $\begin{array}{c}n_{\mathrm{e}}^{\dagger} \\
{\left[10^{4} \mathrm{~cm}^{-3}\right]}\end{array}$ & & \\
\hline NGC 6369 & 16.5 & 1200 & $1.0( \pm 0.1)$ & $0.57( \pm 0.01)$ & $0.82( \pm 0.02)$ & 2 & $0.30( \pm 0.01)$ \\
\hline NGC 6572 & 4.8 & 1060 & $0.8( \pm 0.1)$ & $0.28( \pm 0.01)$ & $10.3( \pm 0.3)$ & 2 & $0.03( \pm 0.01)$ \\
\hline Helix .... & 400. & 213 & $1.0( \pm 0.2)$ & $0.59( \pm 0.03)$ & $0.0126( \pm 0.0005)$ & 2 & $0.39( \pm 0.01)$ \\
\hline NGC 7009 & 12. & 860 & $1.0( \pm 0.3)$ & $0.68( \pm 0.1)$ & $0.84( \pm 0.04)$ & 2 & $0.047( \pm 0.008)$ \\
\hline NGC 6853 & 170. & 264 & $0.8( \pm 0.2)$ & $0.032( \pm 0.001)$ & $2.0( \pm 0.1)$ & 2 & $0.065( \pm 0.006)$ \\
\hline NGC 6720 & 35.5 & 770 & $0.8( \pm 0.2)$ & $0.21( \pm 0.01)$ & $0.47( \pm 0.02)$ & 2 & $0.117( \pm 0.007)$ \\
\hline NGC 6826 & 12.5 & 1200 & $2.2( \pm 0.5)$ & $0.41( \pm 0.02)$ & $0.90( \pm 0.04)$ & 2 & $0.105( \pm 0.005)$ \\
\hline NGC 7027 & 4.5 & 980 & $1.7( \pm 0.3)$ & $0.67( \pm 0.04)$ & $11.5( \pm 0.3)$ & 2 & $0.053( \pm 0.002)$ \\
\hline NGC 6543 & 9.5 & 1000 & $0.9( \pm 0.2)$ & $0.92( \pm 0.01)$ & $1.55( \pm 0.06)$ & 2 & $0.030( \pm 0.002)$ \\
\hline NGC $40 \ldots \ldots$ & 21.5 & 800 & $1.1( \pm 0.5)$ & $0.20( \pm 0.01)$ & $1.27( \pm 0.08)$ & 2 & $0.072( \pm 0.006)$ \\
\hline CRL $618 \ldots \ldots \ldots$ & 0.45 & 900 & $0.36( \pm 0.05)$ & $0.021( \pm 0.001)$ & $9800( \pm 500)$ & 1.3 & $0.00082( \pm 0.00004)$ \\
\hline IC $418 \ldots \ldots \ldots$ & 6.0 & 1000 & $1.3( \pm 0.3)$ & $0.44( \pm 0.02)$ & $5.2( \pm 0.3)$ & 2 & $0.045( \pm 0.003)$ \\
\hline NGC 3242 & 15.5 & 550 & $0.9( \pm 0.2)$ & $0.7_{( \pm 0.1)}$ & $0.77( \pm 0.05)$ & 2 & $0.024( \pm 0.002)$ \\
\hline
\end{tabular}

Notes. ${ }^{(\dagger)}$ Electron density at the inner radius.

Planck Collaboration XXVIII (2014). In order to build the SEDs of the PNe in our sample, the PCCS was queried with a searching radius of $30^{\prime}$ around each $\mathrm{PN}$ position as reported in our master catalogue. We assume a positional coincidence if a Planck source is found within the Planck beam $\left(\theta_{\text {beam }}\right)$, where $\theta_{\text {beam }}$ is function of the channel's frequency.

The PCCS provides multiple estimates of the flux density for each source. In our analysis we use the Detection pipeline photometry (DETFLUX), which assumes that the sources are point-like. As previously described, a typical PN SED consists of two main components, the free-free emission from ionized gas and thermal emission from dust, even though an extra contribution (sometimes called anomalous microwave emission) has been claimed (Casassus et al. 2007). In the case of the LFI channels we expect the emission from PNe to arise essentially from the ionized gas (free-free emission). The dimensions of the associated radio emission are independently known and the PNe can be quite confidently considered as point sources compared to $\theta_{\text {beam }}$. In the case of the HFI channels, because of the lack of knowledge of the extension of the dusty CSEs around our sources, and because of the smaller size of $\theta_{\text {beam }}$ at such frequencies, we perform a posteriori visual inspection of the environment of each detected source in the corresponding Planck map to ensure that the assumption of being point-like holds, and also to evaluate the influence of any confusion from diffuse emission not related to the target source.

In the case of the Helix, which in the Planck maps appears to be quite extended, we prefer to perform non-blind aperture photometry directly on the Planck maps, assuming an aperture with a radius $\left(R_{\mathrm{a}}\right)$ of

$R_{\mathrm{a}}=\sqrt{(F W H M)^{2}+(\Omega)^{2}}$,

where $\Omega$ is the angular size of the Helix, assumed to be 13.4 (O'Dell et al. 2004), and the FWHM at each frequency is the average width as given in Planck HFI Core Team (2011b) and Zacchei et al. (2011). The local background and the uncertainty on the aperture flux are estimated in an annulus with a width of

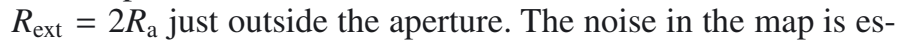
timated from the variance in the outer annulus. Uncertainties in the flux estimation are calculated using the sum in quadrature of the rms of the values in the background annulus plus the absolute calibration uncertainties on each map (Planck Collaboration I 2011).

By definition, all the PCCS detections have $S / N \geq 4$. However, to be sure of the robustness of our detections, we consider only sources detected in at least three different channels. Because of the specific scientific aim of this paper, namely to build and to model the SED in order to obtain insight on the ionized gas and dust components, we impose the further constraint that sources must be detected by both LFI and HFI. Sources PN M1-78, PN A66 77, PP 40 and NGC 2579, which satisfy the above criteria, were removed from the original sample, since they have been misclassified; they are actually compact H II regions (Kohoutek 2001). The thermal dust emission of the PN NGC 6720, the famous Ring Nebula (M 57), was clearly detected in all the HFI channels, except at $100 \mathrm{GHz}$. However, due to the low flux level of the free-free emission, it was not detected by LFI. The source, even though it does not meet the above selection criteria, was nevertheless kept in the sample because of the many ancillary radio data points available from the literature that allow the free-free component to be well defined. Our final sample consists of $13 \mathrm{PNe}$.

Planck flux densities (DETFLUX), as extracted from the PCCS, are reported in Table 1, with their associated uncertainties $(1 \sigma)$. For all the detected sources the DETFLUX flux densities are in good agreement with each other and with ancillary observations.

From Table 1 it is evident that for several sources we do not have detections in all of the Planck channels. This is attributable to a combination of two main effects: PNe are intrinsically weak compared to the sensitivity level of Planck; and they are located preferentially at low Galactic latitudes, where confusion due to diffuse free-free emission and dust prevents accurate photometry. For example, based on the radio flux densities reported in Umana et al. (2008a) and in Pazderska et al. (2009), there are five sources, namely NGC 6302, PN H1-12, NGC 6537, PN M1-51, and NGC 1514, which should have been detected with high $\mathrm{S} / \mathrm{N}$, but have only been detected in one or two channels, these possibly being spurious detections. Among them, four are located very close to the Galactic plane, with 
Planck Collaboration: The millimetre emission from planetary nebulae
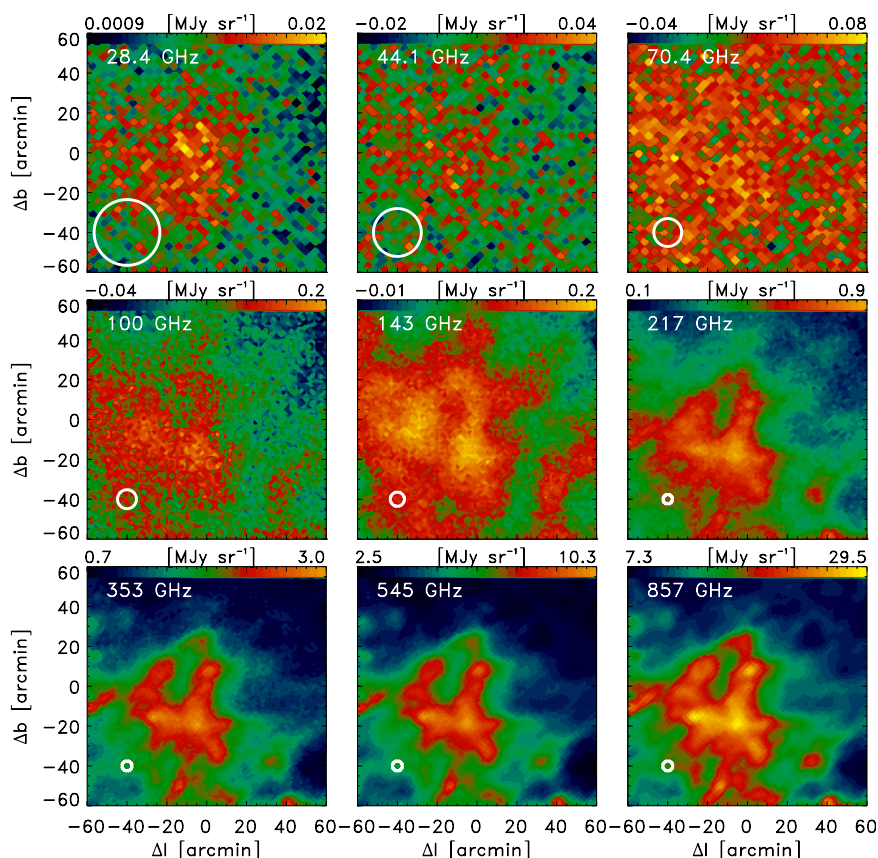

Fig. 1. Planck maps of NGC 1514. The central PN is embedded in a region of diffuse emission that is not clearly related to the source, which prevents us from obtaining a good photometric measurement. The average FWHM of the effective beam is shown in the bottom-left corner of each map.

strong contamination of both diffuse ionized gas and dust. To investigate the missing detection for NGC 1514, which should have a flux of about $900 \mathrm{mJy}$ and is located at high Galactic latitude $\left(b=15^{\circ}\right)$, a direct inspection of the Planck maps was performed. This revealed that the central PN is embedded in a very extended dusty structure preventing a flux measurement (see Fig. 1).

In other cases there are hints of extended dusty haloes around the central PN in the higher-frequency Planck maps (see Figs. 2 and 3), which, however, are still very well detected in several Planck channels, allowing us to accurately measure fluxes. Usually, the effect of such extended emission is to reduce the $\mathrm{S} / \mathrm{N}$. However, in extreme cases, the related flux measurement is disregarded, because from direct inspection of the corresponding Planck map there is strong confusion due to diffuse emission that is not related to the target. As an example, in the case of NGC 6369 the nearby IR structure (see Fig. 2) strongly contaminates the source photometry at $v \gtrsim 300 \mathrm{GHz}$.

To further test the reliability of the Planck data we use the well-known PN NGC 7027, whose radio flux density evolution has been studied by Zijlstra et al. (2008). These authors determined the rate at which the radio flux density is changing, caused by the expansion of the ionized nebula. The Planck measurements of NGC 7027, displayed in Fig. 4, are in very good agreement with the radio spectrum provided by Zijlstra et al. (2008) when evolved to the mean epoch of the Planck measurements, and the sub-millimetre and infrared ancillary data (Telesco \& Harper 1977; Elias et al. 1978; Moseley 1980; Ulich 1981; Gee et al. 1984; Steppe et al. 1988; Hoare et al. 1992; Knapp et al. 1993; Altenhoff et al. 1994; Sandell 1994; Egan et al. 2003; Murakami et al. 2007; Di Francesco et al. 2008).

Before modelling the SEDs, a colour correction has been applied to account for the finite bandpass at each frequency. Since the correction factors depend on the power law of the spectrum inside the band, for each frequency a spectral index
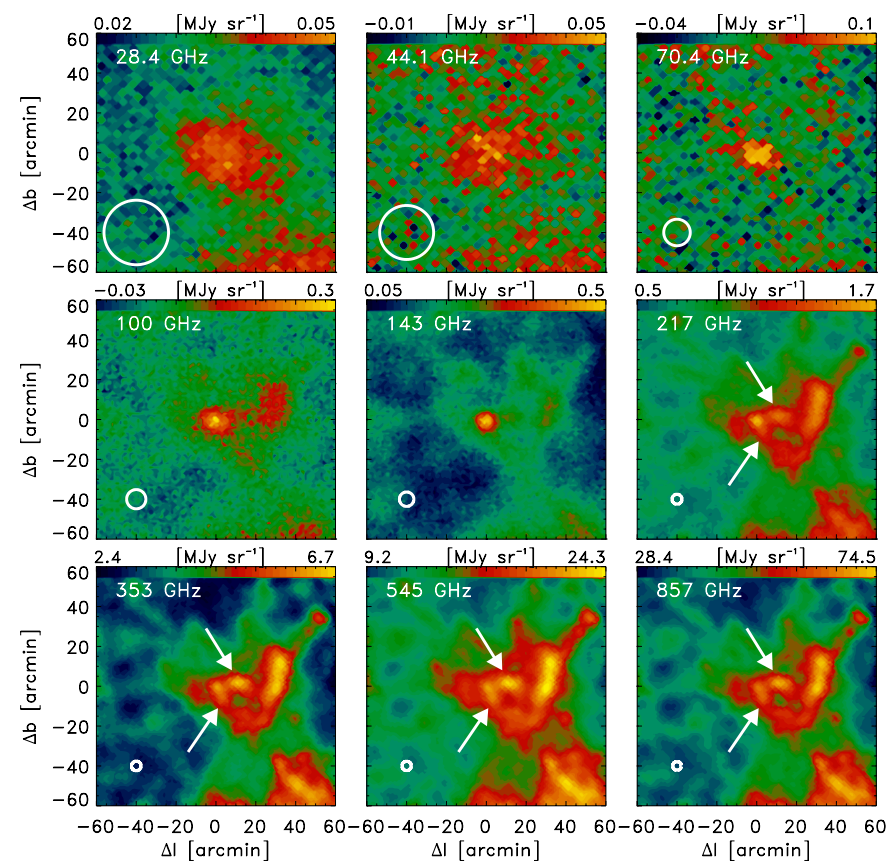

Fig. 2. Planck maps of NGC 6369. An extended structure, marked by the arrows, appears to surround the PN, mostly visible in the HFI channels. This may constitute a hint for the presence of a dusty halo. However, the central component is well detected in several channels, allowing us to calculate its flux. The average FWHM of the effective beam is shown in the bottom-left corner of each map.
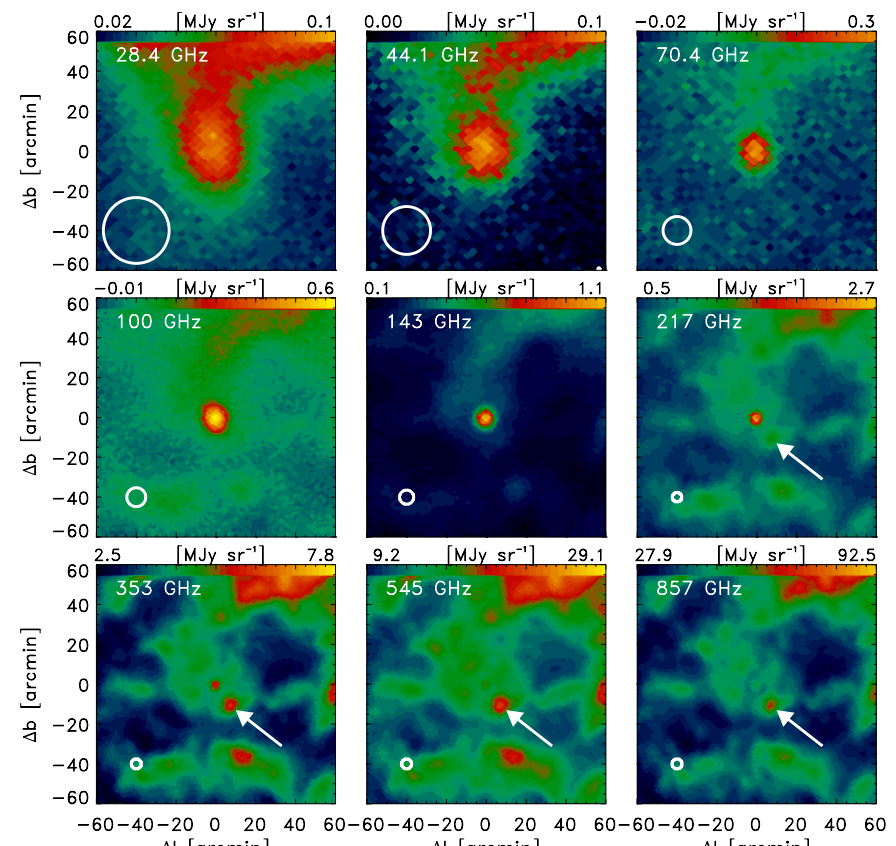

$\Delta l[\operatorname{arcmin}]$

$\Delta l$ [arcmin]

$\Delta l$ [arcmin]

Fig. 3. Planck maps of NGC 7027. Similar to the case of NGC 6369, the central component is embedded in an extended dusty halo, visible in most of the HFI channels. There is also a nearby IR source (marked by the arrow), that is not related to the PN. The average FWHM of the effective beam is shown in the bottom-left corner of each map.

has been computed based on the flux density values reported in Table 1, relative to the adjacent bands. The colour corrections have then been computed following the procedure described in Planck Collaboration II (2014) and Planck Collaboration VI (2014). This is typically of the order of a few times $0.1 \%$ for 


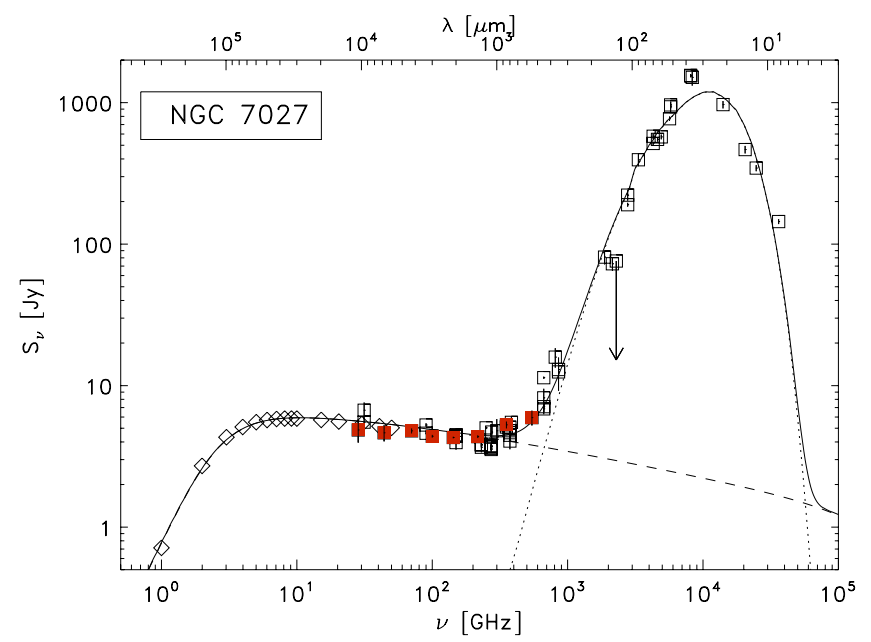

Fig. 4. SED of NGC 7027. Ancillary measurements, obtained with different instruments, are shown as open squares, while the Planck data are shown as red squares. The arrows indicate upper limits. The diamonds show the radio spectrum of NGC 7027 given by Zijlstra et al. (2008) and evolved to the mean Planck observing time. The continuous line is the model of the SED, obtained by combining both free-free (dashed line) and thermal dust emission (dotted line).

LFI channels and up to $7 \%$ for HFI channels, and, as such, it is much lower than the uncertainty associated to the flux density. Only one colour correction iteration was applied. The Planck data points of the selected targets are displayed as red squares in Figs. 4-6 and 9.

\section{Physical properties of the source sample}

In the spectral range between IR and radio, the SED of a PN is characterized by two components: the thermal emission from dust grains; and the free-free emission from the ionized part of the CSE. Recently, it has been realised that dust in and around ionized regions in PNe can play an important role in the energetic output of PNe, and up-to-date photo-ionization codes now include the effect of the dust (van Hoof et al. 2000).

We have first tested different radiative transfer codes that work in dusty environments to identify the most appropriate for our scientific aim. One such code is the photo-ionization code CLOUDY (Ferland et al. 1998). Although a good match is obtained at near-IR wavelengths, in our trials CLOUDY was typically unable to reproduce the long-wavelength spectrum, in particular the optically thick part of the radio continuum, where the spectral slope of the model is typically too steep, implying that radiation transfer is not properly taken into account for the free-free emission. Also, CLOUDY aims at reproducing both continuum and line spectra, therefore UV, optical, and IR spectral data are necessary to constrain its output. Since our goal is to fit only the continuum emission, the use of CLOUDY would introduce unnecessary degrees of freedom in our modelling. Furthermore, those degrees of freedom would be difficult to constrain with a homogeneous set of spectra for our sources: for example, optical spectra for targeted regions within very extended nebulae would certainly not account for the emission from the whole target, which is the case for the other data from IR surveys and single-dish radio telescopes. We thus prefer to model the thermal dust emission with the publicly-available DUSTY code (Ivezic et al. 1999) in combination of our own free-free modelling.

We first model the centimetre continuum radiation with our code for free-free emission (Umana et al. 2008b) used to fit the
SED. This has been improved by implementing a minimization procedure consisting of an iterative process where the free-free emission is calculated within a grid of model parameters, with the ranges and steps gradually restricted until the $\chi^{2}$ stabilizes. The errors associated to the model parameters have been estimated by varying separately each parameter until the $\chi^{2}$ increases of a unit.

Following the wind-shell model, basic geometry usually adopted in the case of the planetary nebulae (Taylor et al. 1987), each source has been modelled as a central cavity surrounded by a shell of ionized gas. The source geometry is characterized by the linear diameter and the ratio between the internal and external radii $\left(r_{\text {int }} / r_{\text {out }}\right)$, where the density distribution of the ionized gas is described by the power law $n_{\mathrm{e}}(r) \propto r^{-\alpha}$. The radio spectrum has been calculated with the ratio $r_{\text {int }} / r_{\text {out }}$, the electron density at $r_{\mathrm{int}}\left(n_{\mathrm{e}}\right)$ and the electron temperature $(T)$ as model parameters.

The radius of the free-free source, the distance and the index $\alpha$ describing the spatial distribution of the ionized gas have been set to fixed values to constrain the model. The slope $\alpha$ was set to 2 , consistent with a spherical wind in steady-state, with the exception of CRL 618 where it was necessary to vary it to assure a good fit to the observed data (see Table 2). The angular diameters of the radio sources have been mainly derived from the NRAO VLA sky survey (NVSS; Condon et al. 1998). In the case of partially resolved sources, this diameter is related to the actual diameter of the source by a shape factor that accounts for the true structure of the source, and a correction has been applied (following van Hoof 2000) to derive the true source size (Umana et al. 2008a).

Distances have been generally adopted from Pottasch \& Bernard-Salas (2010), with exceptions being NGC 6572 (Phillips \& Márquez-Lugo 2011), NGC 6720 (O'Dell et al. 2007), NGC 6853 (Stanghellini et al. 2008), NGC 7009 (Fernández et al. 2004), and NGC 7027 (Zijlstra et al. 2008). The best-fit parameter values are summarized in Table 2 . The total mass of ionized gas has been calculated numerically by integrating the electron density over the entire structure of the radio source. The obtained density and total ionized mass scales with the adopted distance $(D)$ as $\propto D^{-1 / 2}$ and as $\propto D^{5 / 2}$ respectively.

DUSTY modelling was performed after the best-fit value for the radio emission was obtained. General inputs for DUSTY are: the central object radiation field (assumed to be a Planck function with an appropriate temperature and luminosity); composition and size distribution of the dust grains; and a model for the dusty shell (density distribution, optical thickness $\tau_{\mathrm{V}}$ in the visual, geometrical thickness, and temperature of the grains at the inner radius of the shell, $\left.T_{\text {dust }}\right)$. DUSTY assumes spherical symmetry for the dusty envelope. Although such symmetry is not always observed in our targets, DUSTY can still be used to obtain first-order approximations of nebular parameters.

DUSTY allows us to use six different grain types: warm O-deficient silicates (Sil-Ow); cold O-rich silicates (Sil-Oc); astronomical silicates (Sil-DL); graphite (grf-DL); amorphous carbon (am-C); and silicon carbide ( $\mathrm{SiC}-\mathrm{Pg}$ ). To assess the dominant mineralogy of the dust in the circumstellar envelope of our targets (i.e., C-rich versus O-rich), we use the direct information on dust features provided by ISO spectra, namely: Cernicharo et al. (2001) for CRL 618; Bernard Salas et al. (2001) for NGC 7027; Bernard-Salas \& Tielens (2005) for NGC 6543; Volk (2003) for IC 418; Szczerba et al. (2001) for NGC 6369 and NGC 40; Surendiranath \& Pottasch (2008) for NGC 6826; and Phillips et al. (2010) for NGC 7009. In the remaining cases, we use the $\mathrm{C} / \mathrm{O}$ gas-phase abundance (Liu et al. 2001), as this 

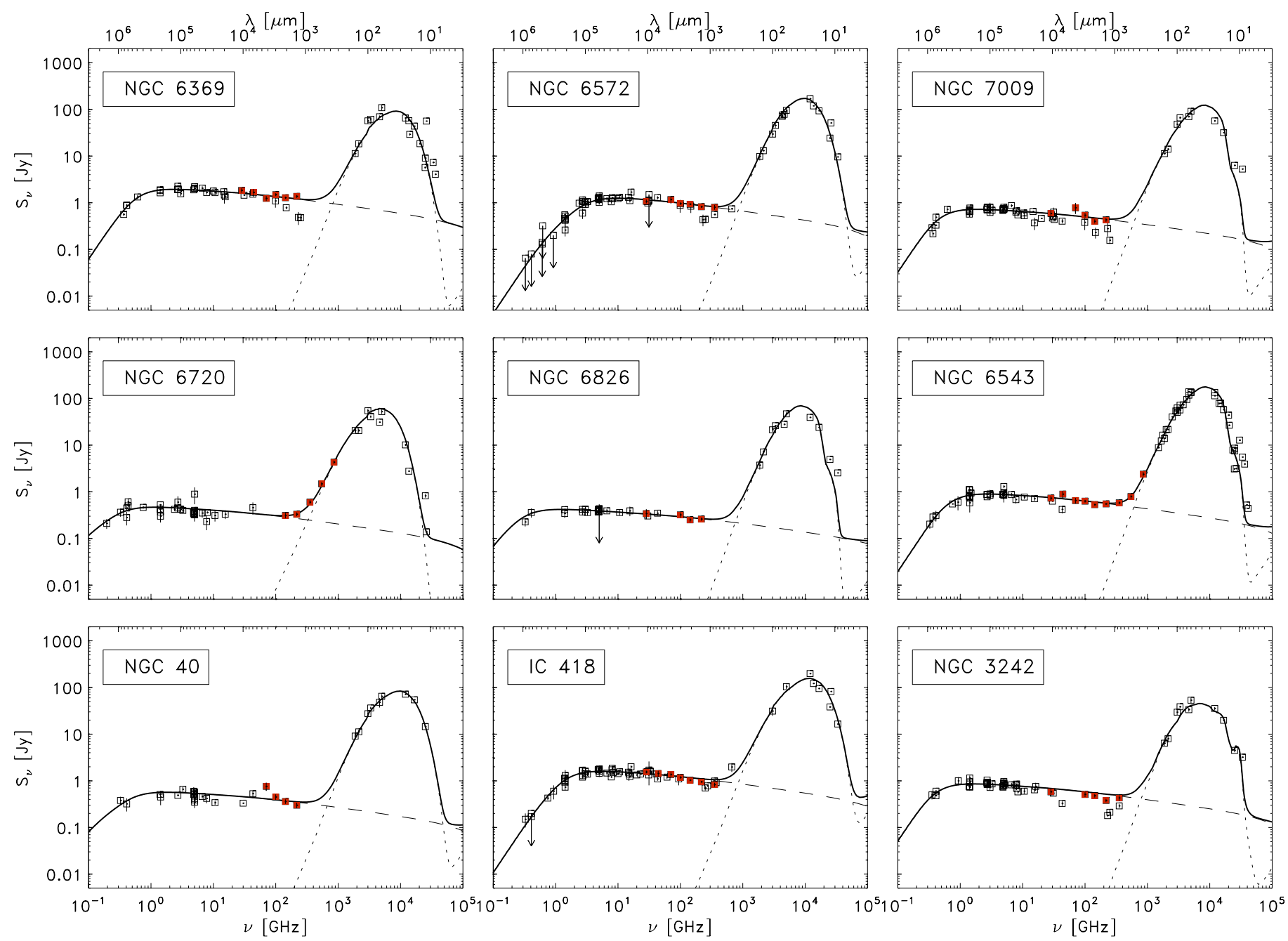

Fig. 5. Models of the SEDs for our sample of Galactic PNe. The continuous line is the combination of both free-free (dashed line) and thermal dust emission (dotted line). Measurements obtained with different instruments and collated from the literature are shown as open squares. Planck measurements are indicated by red squares. The arrows indicate upper limits.

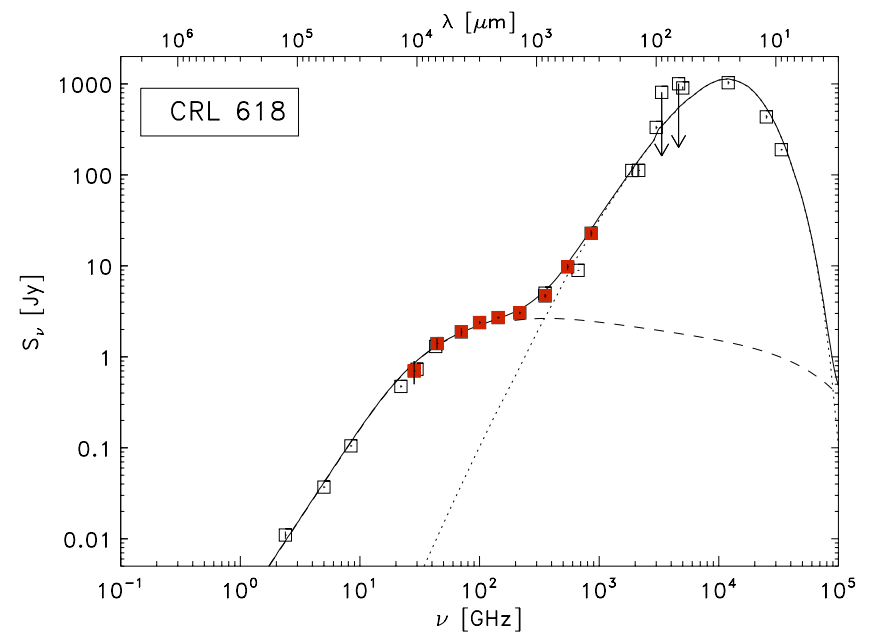

Fig. 6. Model of the SED of CRL 618 (continuous line) considering both free-free (dashed line) and thermal dust emission (dotted line). Ancillary measurements, obtained with different instruments (IRAS, Helou \& Walker 1988; Akari, Murakami et al. 2007), are shown as open squares, while Planck data are shown as red squares. The arrows indicate upper limits. For the ancillary radio measurements $(v \leq 40 \mathrm{GHz})$ only VLA data coeval to Planck observations have been used (see text). is a good indicator of the presence or absence of Carbon-based dust grains (i.e., PAH) in several PNe (Cohen \& Barlow 2005). Furthermore, the choice among different kinds of silicates or Carbon-based grains was based on what provides the best fit to the SED. The standard MRN (Mathis et al. 1977) grain size distribution (grain radius $a=0.005-0.25 \mu \mathrm{m}$, with a distribution following $a^{-3.5}$ ) was applied to all sources.

The density distribution was set to depend on $r^{-2}$ for all sources. Such a distribution follows naturally from the assumption that the circumstellar material was ejected during the AGB phase at a constant mass-loss rate. The only exception is NGC 3242, where a density distribution of $r^{-1.1}$ was necessary to fit the dust contribution. NGC 3242 is not particularly different from the other targets with respect to the optical morphology, evolution, and position, and therefore it is very difficult to justify a different dust distribution in the model. However in this context, it may be worth pointing out that the presence of a system of concentric rings, whose brightness is higher in the mid-IR than in the optical, located in an extended halo around the source, has been recently reported by Phillips et al. (2009). Furthermore, from the analysis of its HST images, Ruiz et al. (2011) modelled its $\mathrm{H} \alpha$ brightness spatial distribution as a thin shell with a constant density embedded within an outer shell, 
characterized by a radial density profile much shallower than the classical profile for a free-expanding wind at a constant massloss rate $\left(r^{-2}\right)$. This is in agreement with our dust modelling for NGC 3242, whose density profile is less steep than that of the other PNe. Interestingly, the radius of the dusty CSE as derived from our DUSTY modelling (about 80") matches quite well with the reported size of the extended halo (about $75^{\prime \prime}$ ) where the concentric dusty rings are located.

We have observed in NGC 7009 and NGC 6826 that the fit underestimates the near- and mid-IR emission, despite taking into account the free-free emission from our radio model. This may point to the presence of a separate population of hot grains $\left(T_{\text {dust }} \geq 500 \mathrm{~K}\right)$. Very little is known about the spatial distribution of dust in PNe. However, given the variety of shapes of PNe observed in the optical and near-IR, the presence of a hotter population of grains may be linked to the specific distribution of dust, with some warmer regions closer to the central star. It is difficult to estimate the amount of such an extra dust component without adding further free parameters to the DUSTY fit. On the other hand, even if such a warm dust component exists, our simulations indicate that it does not have any effects on the temperature of the cooler dust component traced by the millimetre and far-IR emission. The slope of the SED at long wavelengths is determined by the opacity and the size of the dust shell (i.e., the contribution from colder dust). We produced our models taking into account the necessity to properly fit the Planck data and imposing that the outer temperature of the dust shell must match a mean ISM temperature of 10-20 K.

DUSTY treats the problem of radiation transfer independently of the distance to the star by taking advantage of the scaling properties of the radiative transfer equation (Ivezic \& Elitzur 1997). This implies that its output must be scaled to the actual data. We calculated the scaling factor taking into account the estimates of distance and luminosity available in the literature (Goodrich 1991; Sabbadin et al. 2004; Prinja et al. 2007; Gruenwald \& Aleman 2007; Surendiranath \& Pottasch 2008; Zijlstra et al. 2008; Pottasch \& Bernard-Salas 2008, 2010; Morisset \& Georgiev 2009; van Hoof et al. 2010; Monteiro \& Falceta-Gonçalves 2011). Adjustments of both parameters (distance and luminosity) were necessary to match the observational points. From the derived parameters, it is possible to obtain an estimate of the total mass of dust in the shell from the equations derived by Sarkar \& Sahai (2006):

$M_{\text {dust }}=4 \pi R_{\text {int }}^{2} Y\left(\frac{\tau_{500}}{\kappa_{500}}\right)$,

which is valid in the case of an $r^{-2}$ density distribution and

$M_{\text {dust }}=\frac{4 \pi \tau_{500}}{19 \kappa_{500}} R_{\text {int }}^{2} \frac{Y^{1.9}-1}{1-Y^{-0.1}}$,

which is valid for NGC 3242, where the relation $\rho \propto r^{-1.1}$ is required to model the observational data. $Y=R_{\text {out }} / R_{\text {int }}$ is the relative shell thickness, $\tau_{500}$ is the shell optical depth at $500 \mu \mathrm{m}$ (which is an output parameter from DUSTY) and $\kappa_{500}$ is the dust mass absorption coefficient at $500 \mu \mathrm{m} . R_{\text {int }}$ and $R_{\text {out }}$ are here the inner and outer radii of the dusty region, respectively, which do not necessarily match with the inner and outer radii of the ionized shell.

The optical properties of a material can be described with the use of a complex refractive index $m_{\lambda}=\tilde{n}_{\lambda}+\imath \tilde{k}_{\lambda}$, where $\tilde{n}_{\lambda}=$ $\frac{c k}{\omega}$ and $\tilde{k}_{\lambda}=\frac{c \alpha_{\text {abs }}}{2 \omega}$, with $k$ the wave number, $c$ speed of light, $\alpha_{\text {abs }}$ the absorption coefficient, and $\omega$ the frequency times $2 \pi$.
From the chosen set of optical constants (Ossenkopf et al. 1992; Draine \& Lee 1984 or Hanner 1988), we can then calculate the dust absorption coefficient. The dust extinction cross section is defined as $\sigma_{\text {ext }}=\pi a^{2} Q_{\text {ext }}$, where $a$ is the grain radius and $Q_{\text {ext }}$ the extinction efficiency. At sub-mm wavelengths, scattering can be neglected and the extinction is due to absorption alone:

$\sigma_{\mathrm{abs}}=\pi a^{3} \frac{Q_{\mathrm{abs}}}{a}$.

The absorption coefficient per unit volume of dust is defined as

$\kappa_{V}=\frac{\sigma_{\mathrm{abs}}}{V}$,

where $V$ is the average volume of a dust grain. Hence

$\kappa_{V}=\frac{\sigma_{\mathrm{abs}}}{\frac{4}{3} \pi a^{3}}=\frac{3}{4} \frac{Q_{\mathrm{abs}}}{a}$.

An approximation of $Q_{\mathrm{abs}} / a$ as a function of only $\lambda$ can then be found, such that (Andersen et al. 1999)

$\kappa_{V}=\frac{6 \pi}{\lambda} \operatorname{Im}\left[\frac{m_{\lambda}^{2}-1}{m_{\lambda}^{2}+2}\right]$.

Once $\kappa_{V}$ is known, we assume typical values of dust grain density $\rho_{\mathrm{g}}$ of about $3 \mathrm{~g} \mathrm{~cm}^{-3}$ for silicate grains and $2 \mathrm{~g} \mathrm{~cm}^{-3}$ for carbon grains and then calculate the absorption coefficient per unit mass $\kappa_{M}=\kappa_{V} / \rho_{\mathrm{g}}$, which for our sets of optical constants gives us values of $\kappa_{500}$ of $0.98,1.34$, and $2.07 \mathrm{~cm}^{2} \mathrm{~g}^{-1}$, as appropriate for the kinds of silicates and carbon dust adopted in our fits, namely Sil-Ow (Ossenkopf et al. 1992), Sil-DL (Draine \& Lee 1984), and am-C (Hanner 1988), respectively.

The relevant parameters of the modelling are summarized in Table 3, where we report the inner radius, the ratio between the inner radius of the envelope to the radius of the central object $\left(R_{\mathrm{c}}\right)$, the central star luminosity, the temperature of the dust at the inner radius, the relative thickness of the envelope, $\left(R_{\text {out }} / R_{\text {int }}\right)$, the optical depth of the envelope at $0.5 \mu \mathrm{m}\left(\tau_{V}\right)$, the dust composition, and the derived dust mass. The free parameters, given as input of DUSTY, are: $T_{\text {dust }} ; R_{\text {out }} / R_{\text {int }}$; and $\tau_{V}$, the other parameters reported in Table 3 are model outputs or fixed parameters. We should stress here that DUSTY does not have a minimization procedure, therefore the best combination of parameters is obtained through an iterative process consisting of different trials until a good match with the measurements is reached. For NGC 6853 we only report values from the free-free modelling, since no reliable IR ancillary data - necessary to constrain the modelling of the dust contribution - were available.

One major caveat of using DUSTY is that the model considers only a central heating source, neglecting a possible contribution due to the external heating by the interstellar radiation field (ISRF). However, DUSTY allows the modelling of the UV field due to the central star at each point of the circumstellar shell. This field, diluted at the outermost part of the shell, is 4-5 orders of magnitude higher than the value of the ISRF, which is assumed to be $1.6 \times 10^{-3} \mathrm{erg} \mathrm{cm}^{-2} \mathrm{~s}^{-1}$. We thus conclude that the effect of the ISRF is negligible for most of the PNe in our sample. One exception is CRL 618, whose dust mass could be overestimated if the ISRF is excluded, because the optical depth of its shell is very high (see Table 3 ).

As previously mentioned, the DUSTY code assumes spherical symmetry for the dusty envelope. To test the reliability of the physical parameters estimated under this assumption, we look for new sets of parameters that reproduce the observed SEDs, 
Table 3. Source parameters for the DUSTY fit.

\begin{tabular}{|c|c|c|c|c|c|c|c|c|c|c|}
\hline \multirow{2}{*}{\multicolumn{2}{|c|}{ Source }} & \multirow[b]{2}{*}{$\begin{array}{c}R_{\text {int }} \\
{[\operatorname{arcsec}]}\end{array}$} & \multirow[b]{2}{*}{$\begin{array}{c}R_{\mathrm{int}} / R_{\mathrm{c}}^{\dagger \dagger} \\
{\left[10^{4}\right]}\end{array}$} & \multirow[b]{2}{*}{$\begin{array}{c}\text { Luminosity } \\
{\left[10^{3} L_{\odot}\right]}\end{array}$} & \multirow[b]{2}{*}{$\begin{array}{l}T_{\text {star }} \\
{[\mathrm{K}]}\end{array}$} & \multicolumn{2}{|c|}{ Free } & Parameters & \multirow[b]{2}{*}{$\begin{array}{c}\text { Grains } \\
\text { composition }\end{array}$} & \multirow[b]{2}{*}{$\begin{array}{c}M_{\text {dust }} \\
{\left[10^{-4} M_{\odot}\right]}\end{array}$} \\
\hline & & & & & & $\begin{array}{c}T_{\text {dust }} \\
{[\mathrm{K}]}\end{array}$ & $R_{\mathrm{out}} / R_{\mathrm{int}}^{\dagger}$ & $\tau_{\mathrm{V}}$ & & \\
\hline NGC 6369 & & $2.8( \pm 0.2)$ & $200( \pm 20)$ & 3 & 70000 & $140( \pm 4)$ & 100 & $0.030( \pm 0.001)$ & $\mathrm{am}-\mathrm{C}$ & $4( \pm 3)$ \\
\hline NGC 6572 & & $3.7( \pm 0.3)$ & $180( \pm 20)$ & 8.3 & 80000 & $160( \pm 5)$ & 30 & $0.013( \pm 0.001)$ & $\mathrm{am}-\mathrm{C}$ & $0.7( \pm 0.3)$ \\
\hline Helix ... & & $140( \pm 20)$ & $22000( \pm 4000)$ & 0.12 & 110000 & $34( \pm 2)$ & 20 & $0.0085( \pm 0.0001)$ & am-C & $18( \pm 7)$ \\
\hline NGC 7009 & . & $6.0( \pm 0.4)$ & $300( \pm 20)$ & 5 & 80000 & $115( \pm 3)$ & 100 & $0.0029( \pm 0.0001)$ & Sil-DL & $2( \pm 1)$ \\
\hline NGC 6720 & & $4.8( \pm 0.4)$ & $2400( \pm 200)$ & 0.2 & 120000 & $80( \pm 3)$ & 200 & $0.035( \pm 0.002)$ & $\mathrm{am}-\mathrm{C}$ & $11( \pm 5)$ \\
\hline NGC 6826 & . & $2.3( \pm 0.2)$ & $100( \pm 10)$ & 1.64 & 47500 & $120( \pm 5)$ & 30 & $0.030( \pm 0.002)$ & Sil-DL & $1.5( \pm 0.6)$ \\
\hline NGC 7027 & & $2.6( \pm 0.1)$ & $580( \pm 20)$ & 10 & 190000 & $190( \pm 2)$ & 200 & $0.025( \pm 0.004)$ & am-C & $4( \pm 2)$ \\
\hline NGC 6543 & & $4.8( \pm 0.5)$ & $130( \pm 10)$ & 5.2 & 56000 & $120( \pm 5)$ & 100 & $0.012( \pm 0.001)$ & Sil-DL & $6( \pm 3)$ \\
\hline NGC 40 . & $\ldots \ldots$ & $2.3( \pm 0.2)$ & $74( \pm 6)$ & 1.7 & 50000 & $160( \pm 5)$ & 100 & $0.040( \pm 0.002)$ & am-C & $2( \pm 1)$ \\
\hline CRL 618 . & $\ldots \ldots$ & $0.29( \pm 0.03)$ & $2.0( \pm 0.2)$ & 6 & 30000 & $450( \pm 4)$ & 10000 & $50( \pm 4)$ & $\mathrm{am}-\mathrm{C}$ & $4000( \pm 2000)$ \\
\hline IC 418. & & $2.5( \pm 0.3)$ & $20( \pm 2)$ & 10 & 35000 & $200( \pm 8)$ & 200 & $0.040( \pm 0.001)$ & $\mathrm{am}-\mathrm{C}$ & $6( \pm 3)$ \\
\hline NGC 3242 & & $0.8( \pm 0.2)$ & $80( \pm 20)$ & 0.6 & 80000 & $200( \pm 20)$ & 100 & $0.007( \pm 0.001)$ & Sil-Ow & $0.3( \pm 0.2)$ \\
\hline
\end{tabular}

Notes. Am-C indicates amorphous carbon (Hanner 1988), Sil-Ow indicate O-deficient silicates (Ossenkopf et al. 1992), and Sil-DL are astronomical silicates (Draine \& Lee 1984). ${ }^{(\dagger)}$ To estimate the errors on the input and output parameters the DUSTY code has been used by varying arbitrarily this parameter by a factor of two. ${ }^{(\dagger)} R_{\mathrm{c}}$ indicates the radius of the central star.

after setting the ratio of outer to inner radius to the double and to half of our best value for it. This would then tell us how the physical parameters would be affected by assuming that the size of the modelled sphere were twice our best value or half of it. We thus test how the geometry of the source influences the fit and derive ranges of values for the other input $\left(T_{\text {dust }}\right.$ and $\left.\tau_{\mathrm{V}}\right)$ and output (respectively: the inner radius- $R_{\text {int }}$, the ratio of the inner to the stellar radius- $R_{\text {int }} / R_{\mathrm{c}}$, and the mass of dust- $\left.M_{\text {dust }}\right)$ parameters. As expected, for the output parameter $M_{\text {dust }}$ we have found a variation of about $50 \%$, in agreement with the adopted range of shell thickness. The ranges of the other model parameters thus estimated are instead not very large: $R_{\text {int }}, R_{\text {int }} / R_{\mathrm{c}}$ and $\tau_{\mathrm{V}}$ vary within about $10 \%$, and $T_{\text {dust }}$ less than $5 \%$, on average. This confirms the goodness of our nebular parameters as a first-order estimation. These ranges are reported in Table 3 as errors associated to the DUSTY parameters.

Results for the SEDs, considering both thermal dust and ionized gas contributions, are shown in Figs. 4 and 5.

Planck measurements have provided good spectral coverage of the PN SEDs, in particular at those frequencies where freefree and thermal dust emission contributions overlap. Some authors (Hoare et al. 1992) have investigated the possible presence of cool $(\sim 20 \mathrm{~K})$ dust in the CSEs of PNe. The presence of such a component can be excluded for NGC 6720 and NGC 6543 directly from Planck data, which nicely follow the combination between free-free and thermal dust emission. However, this result can be extended to the whole sample, since in all cases the SEDs can be explained only in terms of free-free and warm thermal dust emission.

For three sources, namely NGC 6369, NGC 7009, and NGC 3242, the high frequency Planck measurements appear to be systematically higher than the ancillary data. However, all the millimetre ancillary data for those sources are from the same study (Casassus et al. 2007), where $31 \mathrm{GHz}$ and 230 plus $250 \mathrm{GHz}$ fluxes are provided, which may suffer from some effects due to the fact that the dusty envelope is partially resolved. Supporting this conclusion, recent LABOCA/APEX measurements (Cerrigone et al., in prep.) of NGC 7009 and IC 418 are in good agreement with those obtained from Planck.
Finally, one of our sources, NGC 40, appears to have a higher emission with respect to the model at around $70 \mathrm{GHz}$. With the current data it is difficult to estimate whether this is related to background contamination and/or to an extra contribution (i.e. anomalous emission). It is worthwhile noting that the presence of strong PAH emission has been reported in NGC 40 (Ramos-Larios et al. 2011), which might be linked to radiation of very small spinning dust grains.

Typical densities and temperatures in the ionized regions are in agreement with those that are generally observed (Buckley \& Schneider 1995). The derived values of total ionized masses are also in agreement with values reported in the literature, which are of the order of a few times $0.01 M_{\odot}$. Besides the extreme cases of CRL 618 and the Helix, which will be analysed separately in the following sections, the dust temperatures obtained are in the range $80-200 \mathrm{~K}$ (Table 3 ), in agreement with previous works. In particular, very close values have been obtained for NGC 40 (Ramos-Larios et al. 2011) and for NGC 7009 (Phillips et al. 2010) from the analysis of Spitzer data. We also confirm the general evolutionary trend in grain temperature observed by other authors on the basis of IR data (Phillips \& Márquez-Lugo 2011), where more evolved PNe (i.e., NGC 6543 and NGC 6720) display lower values. In general, the inner radius of the dusty envelope appears to be coincident with or located inside the ionized region. This leads to the conclusion that ionized gas and dust spatially coexist in these sources, despite the different physical conditions in which these components are presumed to survive. This points to the presence of a possible shielding effect, allowing the dust to survive in the harsh environment of the UV radiation field of the central star.

The total dust mass, as derived from our modelling, is in the range of values reported by Gurzadyan (1997), and, in general, two orders of magnitude smaller than that of the ionized gas mass, but it is distributed in a much more extended spatial region. However, such values of $M_{\text {dust }}$ have to be considered as lower limits. The data collected in this paper mainly trace dust at around $100 \mathrm{~K}$ while our modelling pointed out in several cases the possible presence of an extra component of warmer dust, whose contribution cannot be estimated from this data set. 
Table 4. Continuum flux density for CRL 618 from the CALSUR program (2009-VLA D configuration).

\begin{tabular}{ccccc}
\hline \hline Frequency $\ldots \ldots$ & $5 \mathrm{GHz}$ & $8.3 \mathrm{GHz}$ & $22 \mathrm{GHz}$ & $43 \mathrm{GHz}$ \\
$\ldots \ldots \ldots \ldots \ldots$ & $(C$-band $)$ & $(X$-band $)$ & $(K$-band $)$ & $(Q$-band $)$ \\
\hline Flux density $[\mathrm{mJy}]$ & $37 \pm 0.9$ & $105 \pm 2.0$ & $473 \pm 15$ & $1300 \pm 40$ \\
\hline
\end{tabular}

\section{The proto planetary nebula (PPN) CRL 618}

One of our targets is CRL 618, a PPN that entered the postAGB stage about 200 years ago and is rapidly evolving towards a PN. It provides a unique opportunity to study the physical processes taking place immediately before the birth of a PN. Multiwavelength, multi-epoch observations (IR, radio, millimetre, and optical) have provided us with a complex picture of the source (Sánchez Contreras 2004), consisting of:

1) a torus-like dusty structure located in the equatorial plane and obscuring the central object;

2) two shock-excited optical lobes, extending in the polar axis of the star;

3) a molecular envelope, composed of the AGB remnant CSE plus a fast bipolar outflow;

4) a compact $\mathrm{H}$ II region close to the central object, indicating the onset of ionization of the envelope.

Although numerous observational and theoretical efforts have been made to understand CRL 618, several interesting puzzles remain. Among them is the free-free emission from the central H II region, whose variability has been reported by several authors (Kwok \& Feldman 1981; Sánchez Contreras \& Sahai 2004; Wyrowski et al. 2003). This somewhat controversial behaviour, implyies that CRL 618 exhibits the activity of a post-AGB wind, which is poorly understood (García-Segura et al. 2005).

In order to study the evolution of the radio emission, we have analysed multi-frequency radio data obtained in different epochs and we have concluded that the increase of the radio emission, the derived ionized mass, and the size of the radio nebula are consistent with the hypothesis (Kwok \& Bignell 1984) of the expansion of an ionization front caused by an increase in central star temperature and a larger output of UV photons. Similar conclusions were independently achieved by Tafoya et al. (2013).

In this framework, it is evident how important it is to build the observed SED from almost coeval measurements for this strongly variable source, especially in the millimetre range, where the strongest variability has been reported (Sánchez Contreras et al. 2004). Planck provides for the first time a coverage of the source spectrum in a very poorly explored spectral region, allowing us to derive information on the physical properties of the associated circumstellar envelope without suffering any variability effects.

Assuming that the variability is mainly related to the evolution of the central $\mathrm{HII}$ region, and therefore that it will mostly influence the free-free contribution, we have retrieved from the Karl G. Jansky Very Large Array (VLA) archive the data sets observed as close in time as possible to the nominal Planck mission, defined as 12 August 2009 through 27 November 2010. We found multi-frequency, D-configuration data (Program: CALSUR), obtained in November 2009. Data reduction and imaging were performed by using the software package CASA 3.0.0, following standard procedures. The derived flux density and related rms noise are summarized for each frequency in Table 4. Sánchez Contreras et al. (2004) reports strong variability in the millimetre range. Although continuous monitoring of the millimetric flux density would be necessary for a full characterization of its behaviour, the authors concluded that the millimetric light curve is more complicated than a steady monotonic function and consists of a combination of epochs with increasing and decreasing flux. Such variations have a characteristic time scale of the order of 2-3 years (Sánchez Contreras et al. 2004). On the other hand, from Umana et al. (in prep.), a steady increase of the flux density is observed between about 1 and $20 \mathrm{GHz}$ over a large temporal baseline spanning more than 20 years. At $15 \mathrm{GHz}$ an increase of around $30 \%$ is observed in 10 years. Therefore, even if the VLA and Planck measurements are not exactly simultaneous, we do not expect any significant variation over the one year time between them. This conclusion is also supported by the agreement between the $43 \mathrm{GHz}$ VLA measurement (Table 4) and the $44 \mathrm{GHz}$ Planck measurement (Table 1).

To model the SED, we followed the same procedure as described in the previous section. The size of the radio source was set by following the results from Umana et al. (in prep.), who measured a total extension of $0 .{ }^{\prime \prime} 9$. The results of our modelling of both the free-free and thermal dust emission are shown in Fig. 6, where a clear thermal dust contribution is superimposed on a typical free-free spectrum with a turnover frequency of around $40 \mathrm{GHz}$. The assumed gradient in the density distribution, necessary to fit the observed data, is consistent with the stellar wind model of Martin-Pintado et al. (1988), even though the index of the power law describing the density distribution ( $\alpha=1.3$ ) indicates we are in the presence of something different from an isotropic canonical stellar-wind (Taylor et al. 1987). In the optically thin part of the spectrum, an increasing contribution from thermal dust is evident. This is consistent with results reported by Nakashima et al. (2007), whose SMA observations pointed out the presence at $690 \mathrm{GHz}$ of an extended component partially resolved by the $1 . \prime 0 \times 0$ ". 8 synthetic beam of the interferometer and therefore morphologically different from the compact free-free component. All the relevant parameters of the free-free and DUSTY fits are summarized in Tables 2 and 3.

The physical characteristics of the envelope surrounding CRL 618 stand out with respect to those of the other targets. This is consistent with CRL 618 being a very compact (high $n_{\mathrm{e}}$ ) young PN (or a PPN), where the ionization has just started (a very small value for $M_{\text {ion }}$ ). The compact PPN is still embedded in an extended dusty environment-a remnant of the previous evolutionary stage-containing at least $0.4 M_{\odot}$ of dust. This value of the dust mass in the CSE of CRL 618 is comparable to that derived for the mass of the molecular gas (Sánchez Contreras et al. 2004), estimated as $0.7 M_{\odot}$.

\section{The Helix}

\subsection{Overall picture}

NGC 7293, the Helix, is one of the closest (213 pc; Harris et al. 1997) bright and evolved PNe. Its central star is well resolved from the surrounding nebula and is already on the white dwarf (WD) cooling track, with a temperature of about $1.1 \times 10^{5} \mathrm{~K}$. Thanks to its proximity, several authors have studied the Helix in different spectral ranges and at different spatial scales (Speck et al. 2002; O'Dell et al. 2004; Hora et al. 2006; Meaburn et al. 2013). All together, these observations have provided us with a detailed picture of this evolved nebula, revealing both its highly inhomogeneous nature and its overall large-scale structure. The main ring consists of at least two different emission components 
Planck Collaboration: The millimetre emission from planetary nebulae
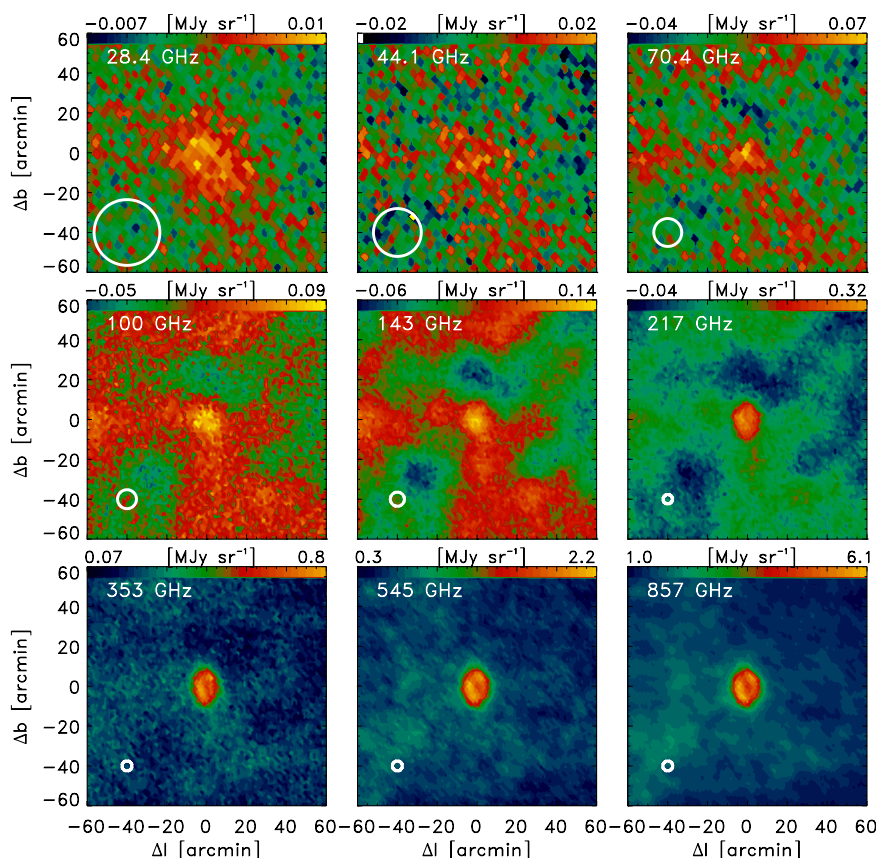

Fig. 7. Planck maps of the Helix (in Galactic coordinates). At each frequency, the corresponding beam (FWHM) is indicated in the bottomleft corner. In addition to the well known structures, associated with the evolved PN, diffuse emission is also present up to one degree from the central object.

with a highly structured texture at a separation of about 4.'2-the so-called cometary knots. An outer ring extends up to $25^{\prime}$ from the central star. The outer ring/disk is flattened on the side colliding with the ambient interstellar medium. For a complete census of all the structures identified in the Helix see O'Dell et al. (2004). Evidence for extended emission was also reported by Speck et al. (2002) in both $\mathrm{H}_{\alpha}$ and far-IR, indicating the presence of a large halo consisting of ionized gas and dust. Such large halo is clearly visible also in the ultraviolet image of the Helix obtained with the GALEX satellite (Zhang et al. 2012; Meaburn et al. 2013). Furthermore, the Helix image performed at ultraviolet wavelengths reveals the existence of a very outer and faint extended halo (diameter up to $40^{\prime}$ ), bow shaped on the east side and jet-like shaped on the west side. These features are oriented along the proper motion direction and were firstly identified in Helix images obtained in the $\mathrm{H}_{\alpha}$ and [N II] $6584 \AA$ emission lines (Meaburn et al. 2005).

The Helix was detected in all Planck channels. The LFI channels are dominated by the ionized gas emission, while starting from $100 \mathrm{GHz}$ an increasing contribution from thermal dust emission is evident, with a ring structure very similar to the main ring observed at optical and infrared wavelengths (Fig. 7). For comparison, the 60 and $100 \mu \mathrm{m}$ IRIS maps are also shown in Fig. 8, where the same overall morphology as observed in the Planck maps is evident. Apart from the well known structures associated with the evolved PN, diffuse emission is also present up to one degree from the central object. Such structures are evident from $100 \mathrm{GHz}$ to $353 \mathrm{GHz}$ and are very probably due to dust. To model the SED of the Helix, we followed the same procedure as described in Sect. 4 for the other sources. Aperture photometry was performed, as described in Sect. 3, on both Planck and IRIS maps. A colour correction was also applied to the Planck fluxes. Only IRIS maps at 60 and $100 \mu \mathrm{m}$ were considered, as they are free from contamination from line emission (Speck et al. 2002)
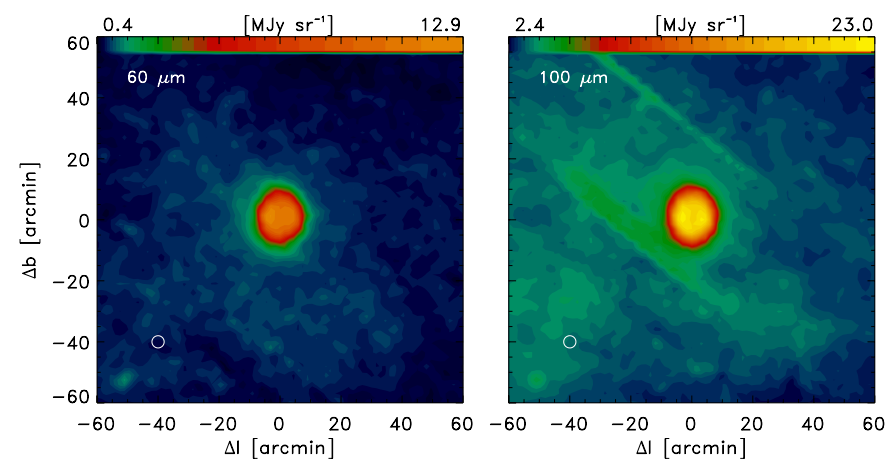

Fig. 8. IRIS 60 and $100 \mu$ m,clip maps of the Helix (in Galactic coordinates). At each frequency, the corresponding beam (FWHM) is indicated in the bottom-left corner.

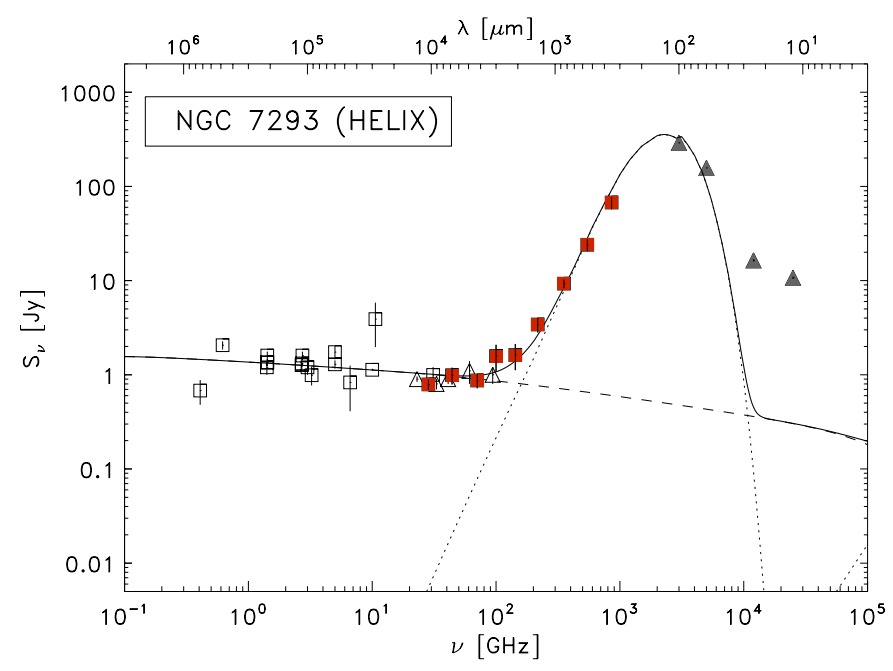

Fig. 9. Model of the SED of NGC 7293 (continuous line) considering both free-free (dashed line) and thermal dust emission (dotted line). Ancillary radio measurements, obtained with different instruments, are shown as open squares (reference numbers given in Table 1: 2, 3, 6, 7, $16,17,18,19,23,29$ and 52), WMAP 7-year data as open triangles, aperture photometry from IRIS as filled triangles, and Planck data are shown as red squares. IRIS data at $12 \mu \mathrm{m}$ and $25 \mu \mathrm{m}$, which are contaminated by emission lines, are also shown in the plot for completeness, but were not considered in the fitting procedure (see text).

and should trace only the dust continuum. The size of the radio source was set to $400^{\prime \prime}(6.7)$, as derived from the cuts performed across the radio source by Rodríguez et al. (2002). All the relevant parameters of the free-free and DUSTY fits are summarized in Tables 2 and 3. Encouragingly, our minimizing procedure provides an internal radius of the radio source (about 240") that is in good agreement with Rodríguez et al. (2002). The results from our modelling of both free-free and thermal dust emission are shown in Fig. 9, where a clear thermal dust contribution is superimposed on a typical optically-thin free-free spectrum. Planck data agree well with data from the WMAP 7-year catalogue and with the $31 \mathrm{GHz}$ data point from Casassus et al. (2004). However, the Planck data are fundamental to constrain the freefree and dust contributions, filling for the first time the observational range between 10 and $10^{3} \mathrm{GHz}$ where the lack of measurements has led to many speculations (Casassus et al. 2004). As in the case of CRL 618, the physical properties of the Helix, as derived from our fit, stand out with respect to the other targets. 
This is a consequence of its evolutionary stage, since the Helix is the most evolved PN of the sample; it is very extended (low $n_{\mathrm{e}}$ ), almost completely ionized (a high value of $M_{\text {ion }}$ ), and embedded in an envelope of mostly low temperature dust.

\subsection{The morphology of the dust component}

Due to the large angular extent on the sky (up to $14^{\prime}$ radius) and the angular resolution of Planck at HFI frequencies (down to $5^{\prime}$ ), it is possible to attempt a morphological study of the outer structures observed in the Helix directly from the Planck maps.

The dust component of the Helix was already observed in the mid-IR and far-IR using ISO and Spitzer (Cox et al. 1998; Speck et al. 2002; Hora et al. 2004). One of the major outcomes from such studies was the presence of a very peculiar size distribution of the dust grains, with a lack of small molecular-sized dust particles, which were probably destroyed by the strong radiation field of the central star (Cox et al. 1998). As a consequence, the ISO and IRAC (Fazio et al. 2004) maps are mainly tracing $\mathrm{H}_{2}$ and fine-structure atomic lines. The only information on the spatial distribution of the dust continuum comes from linear cuts performed on 90 and $160 \mu \mathrm{m}$ ISOPHOT images by Speck et al. (2002) at one particular position of the nebula $\left(\mathrm{PA}=155^{\circ}\right)$, with an angular resolution (FWHM) of 44."5 and 97."4, respectively. The HFI maps, even with their low angular resolution, allow us for the first time to analyse the full spatial distribution of the dust component and to perform a comparative study of the different components coexisting in the nebula (ionized gas, thermal dust, and molecular gas) for a proper modelling of its physics.

We first check if the results from the Planck maps are consistent with the ISO linear cuts (Speck et al. 2002). To do this, we performed cuts on the $217,353,545$, and $857 \mathrm{GHz}$ Planck maps across the entire dust nebula at the same position angle (PA) of $155^{\circ}$ east to north, equatorial, as in Speck et al. (2002). The 217, 353, 545, and $857 \mathrm{GHz}$ profiles are shown in Fig. 10, together with the $160 \mu \mathrm{m}$ profile adapted from Speck et al. (2002). The overall dust distribution agrees well with the ISOPHOT linear scan, indicating that in both Planck and ISOPHOT profiles there is evidence of low-brightness dust emission out to a distance of about $18^{\prime}$ from the central star. The lack of emission from the centre reported by Speck et al. (2002) in the $160 \mu \mathrm{m}$ data, which they interpreted as evidence of a lack of small dust grains, is less evident in the Planck cut at $857 \mathrm{GHz}$, but this can be ascribed to the lower angular resolution $\left(1.6\right.$ versus about $\left.5^{\prime}\right)$.

Close-ups of the 545 and $857 \mathrm{GHz}$ Planck maps are shown in Fig. 11. The high-frequency maps have a quite similar morphology, indicating that they are tracing the same dust component. In particular, the $857 \mathrm{GHz}$ image provides more details on the dust distribution, which can be summarized as follows.

a) An inner, low-brightness region extending up to $5^{\prime}$.

b) A main ring of emission extending up to about $10^{\prime}$ from the central object. Here the dust is not uniformly distributed, but it appears more concentrated in the north-east than in the south-west part.

c) An extended, low-brightness structure extending up to $20^{\prime}$ from the central object in the north-east part of the nebula. Such extended structure is also visible at $545 \mathrm{GHz}$, but merges with the ISM at other frequencies.

All these structures can also be seen in Fig. 12, which shows cuts of the $217,353,545$, and $857 \mathrm{GHz}$ maps along $\mathrm{PA}=235^{\circ}$, where the minus side of the cuts correspond to the left-hand side of

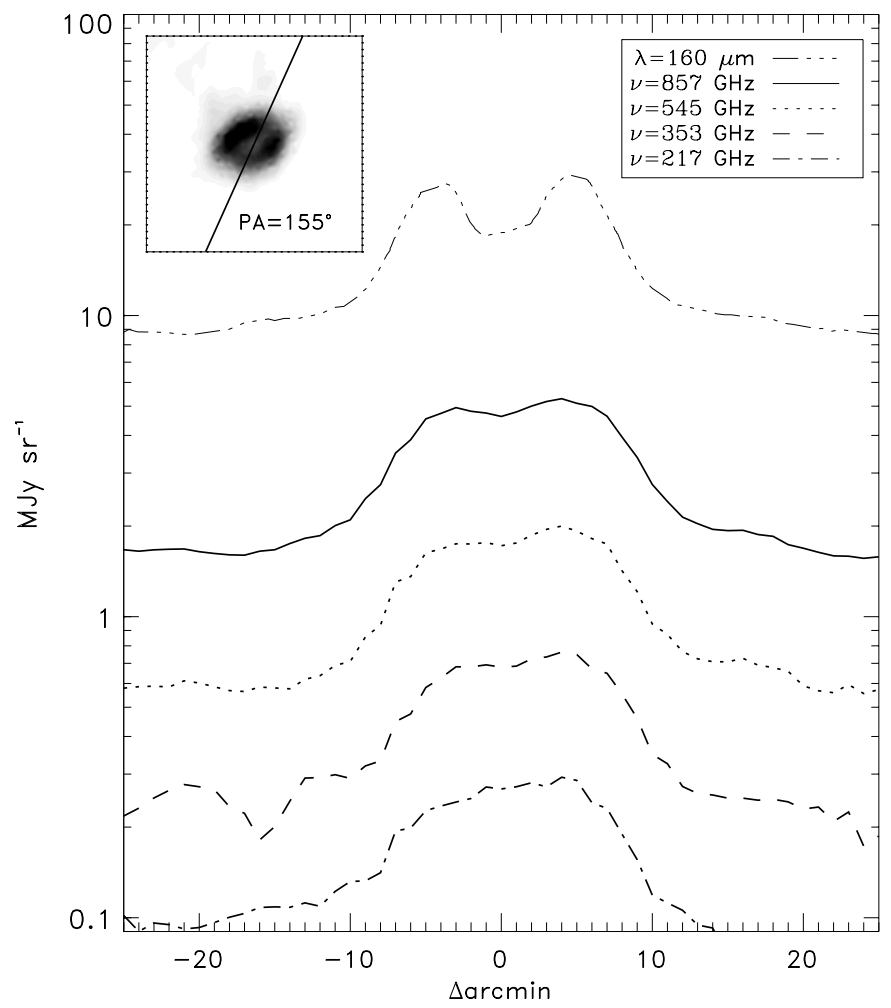

Fig. 10. Profiles obtained from the Planck maps of the Helix at 217 (dash dot line), 353 (dashed line), 545 (dotted line), and $857 \mathrm{GHz}$ (continuous line), with cuts at the same PA as in Speck et al. (2002). The direction along which the cuts were performed is indicated in the small inset on the top left (superimposed on the $857 \mathrm{GHz}$ Planck map, in equatorial coordinates). The Planck profiles are compared to the $160 \mu \mathrm{m}$ profile reported in Speck et al. (2002) (triple dot dashed line).

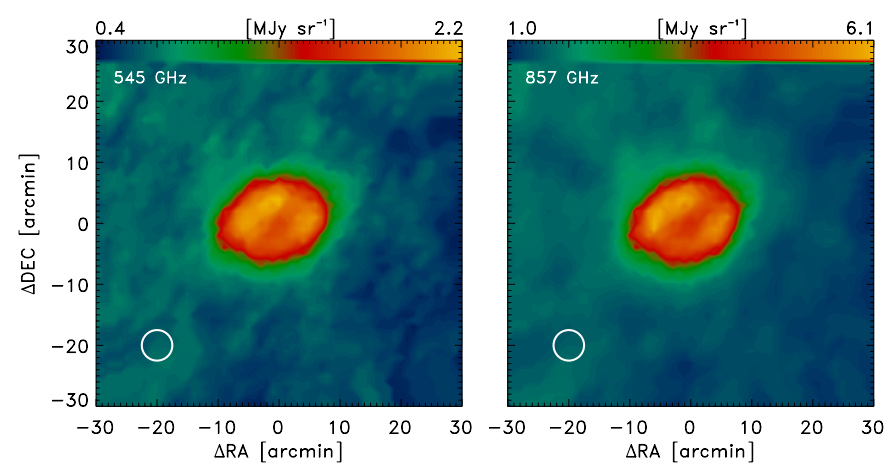

Fig. 11. Close-ups of the Planck maps of the Helix (in equatorial coordinates) at 545 (left) and $857 \mathrm{GHz}($ right).

the inset map. While the presence of an inner cavity was already pointed out in ISO observations and interpreted in terms of small dust grains being rapidly destroyed by the strong UV field of the central star (Cox et al. 1998; Speck et al. 2002), the presence of extended low-brightness structures is a unique result of the present observations. A hint of its presence was pointed out from linear cuts performed with ISOPHOT by Speck et al. (2002), but only Planck has provided us with the first (even though at low-resolution) mapping of this dusty structure, revealing its axisymmetric morphology, which is more extended toward the north-east. Such extended low-brightness dusty emission seems spatially associated with the north-east outer arc clearly visible 
Planck Collaboration: The millimetre emission from planetary nebulae

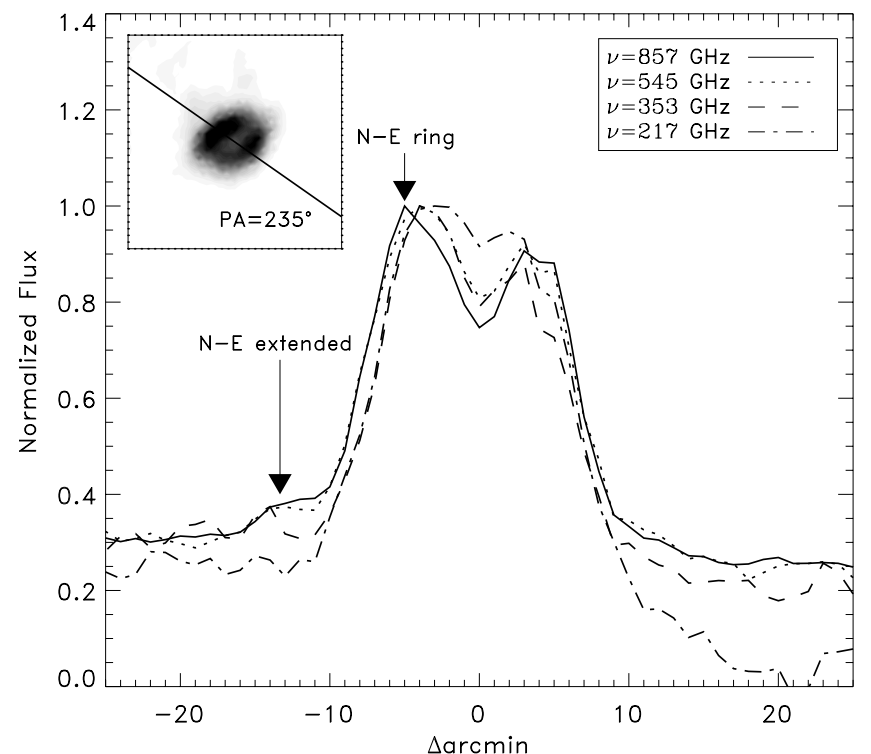

Fig. 12. Profiles of linear scans performed in the Planck maps at 217 (dash dot line), 353 (dashed line), 545 (dotted line) and $857 \mathrm{GHz}$ (continuous line) normalized to the $217 \mathrm{GHz}$ profile, to better compare them. The direction along which the cuts have been performed is indicated in the small inset on the top left. It is superimposed to the $857 \mathrm{GHz}$ Planck map $\left(\mathrm{PA}=235^{\circ}\right)$ and has been chosen in order to include the extended structure.

in the optical and ultraviolet images. Interestingly, Zhang et al. (2012) recently reported the discovery of a mid-IR halo around the Helix observed with the Wide-field Infrared Survey Explorer (WISE) at $12 \mu \mathrm{m}$, whose dimensions and morphology are very similar to those observed in the extended Planck structure. As the mid-IR halo was not detected at $22 \mu \mathrm{m}$ and marginally detected by WISE at $3.4 \mu \mathrm{m}$ and $4.6 \mu \mathrm{m}$, Zhang et al. (2012) concluded that its emission peaks at $12 \mu \mathrm{m}$ and attributed the emission to a warm ( $T_{\text {dust }}$ about $300 \mathrm{~K}$ ) dust component. However, the broader emission observed in the HFI channel implies that there is also a contribution from colder dust.

It is not possible to directly compare our results from the DUSTY fitting with the morphological information provided by Planck maps. In fact, while there is an overall agreement between the inner radius for dust distribution, the value of the total extension of the dusty CSE provided by DUSTY corresponds to regions where the dust reaches the temperature of the ISM, which is assumed to be $12 \mathrm{~K}$ in the modelling. The thermal emission from such cold dust will be very weak at the Planck and IRAS frequencies, which are mainly tracing warmer dust.

It is interesting to analyse how the dust component observed by Planck is morphologically related to the molecular and ionized gas component. To do so, we compare the Planck $857 \mathrm{GHz}$ map to the ground-based near-IR molecular Hydrogen $(2.12 \mu \mathrm{m})$ image from Hora et al. (2004) and to the radio map at $1.4 \mathrm{GHz}$ from the NVSS. In the $\mathrm{H}_{2}$ map, the well-known double ring structure is clearly seen (see Fig. 13) and is very similar to that observed in the optical image. Before making a direct comparison of the two maps, we subtracted the very bright emission from field stars in the $\mathrm{H}_{2}$ image and then convolved it with the Planck beam at $857 \mathrm{GHz}$. The comparison between the two components is shown in the right-hand panel of Fig. 13.

The molecular component is spatially confined within the dust component (only the main ring of dust emission has been reproduced in the image). However, we cannot exclude the presence of $\mathrm{H}_{2}$ beyond the dust emitting region, since at this distance
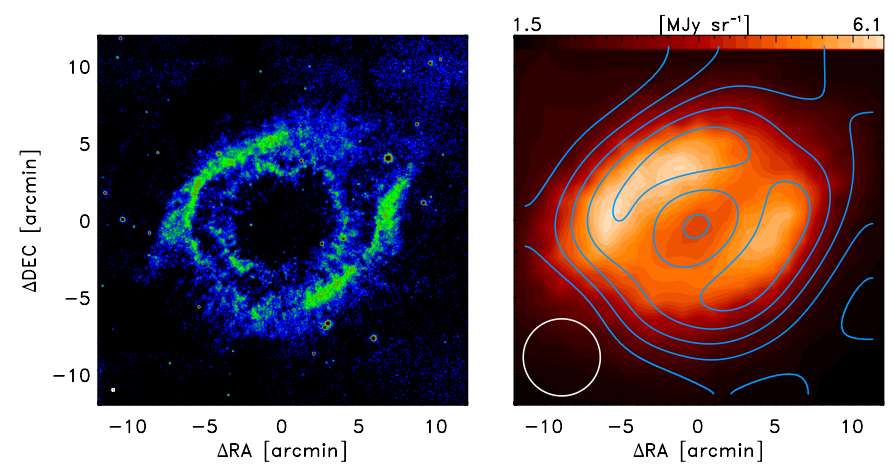

Fig. 13. Left: $\mathrm{H}_{2}$ map of the Helix (Hora et al. 2004). Right: the comparison between the $\mathrm{H}_{2}$ map (contours) and the Planck $857 \mathrm{GHz}$ map. Only the main-ring emission of the dust component has been reproduced. The $\mathrm{H}_{2}$ map has been smoothed to the angular resolution of the Planck map, indicated in the left-bottom corner.

from the central star $\mathrm{H}_{2}$ is not excited, and therefore, even if present, it cannot be detected (Aleman et al. 2011).

In order to study the morphology of the ionized gas, we retrieved a radio image of the Helix at $1.4 \mathrm{GHz}$ from the NVSS web archive ${ }^{4}$. The radio morphology is the same as in Rodríguez et al. (2002), where there are several point sources in the field that are not related to the nebula, but are probably extragalactic sources. To better determine the relative distributions of each component in the nebula, profiles have been obtained with cuts performed at two set position angles in the radio (NVSS), dust (Planck), and $\mathrm{H}_{2}$ maps (Fig. 14). The maps have first been smoothed to the angular resolution of the Planck $857 \mathrm{GHz}$ map. The radio map traces the inner ring, where most of the radio emission comes from, surrounded by both the molecular $\left(\mathrm{H}_{2}\right)$ and dust (Planck maps) components.

NVSS observations, even though taken at $1.4 \mathrm{GHz}$ with the VLA in its most compact configuration (typical synthesized beam of the order of $45^{\prime \prime}$ ), are not sensitive to structures larger than around $15^{\prime}$. Therefore, in the NVSS map, very extended low-brightness structures, such as those observed in $\mathrm{H}_{\alpha}$ and reported by Speck et al. (2002), might be resolved out. Rodríguez et al. (2002) pointed out that the ionized gas, as traced by the continuum radio observations, is surrounded by $\mathrm{HI}$ emission $(21 \mathrm{~cm}$ line $)$ as indicated by the observations carried out with a similar instrumental configuration. The H I distribution well reproduces the typical helical structure of the molecular envelope, with a morphological similarity with the $\mathrm{H}_{2}$ emission, and is actually delineating the outer parts of the bright ringlike $\mathrm{H}_{2}$ region. The ring-like structure of the inner ionized gas versus the more extended morphology of the molecular envelope is also supported by the images obtained using ISOCAM with the LW2 filter (centred at $6.9 \mu \mathrm{m}$ ) and the LW3 filter (centred at $15 \mu \mathrm{m}$ ) by Cox et al. (1998). Again, while the former traces the $\mathrm{H}_{2}$ emission and thus delineates the molecular envelope morphology with the classical helical structure, the latter is dominated by the [Ne III] emission and thus traces the ionized gas, clearly showing a more compact, rounder morphology. The interplay between the dust and ionized components is likely to evolve with the nebular expansion (Chu et al. 2009). At the angular resolution of Planck, our data indicate that the ionized material is concentrated within the dusty nebula, closer to the central star.

4 http://www.cv.nrao.edu/nvss/postage.shtml 

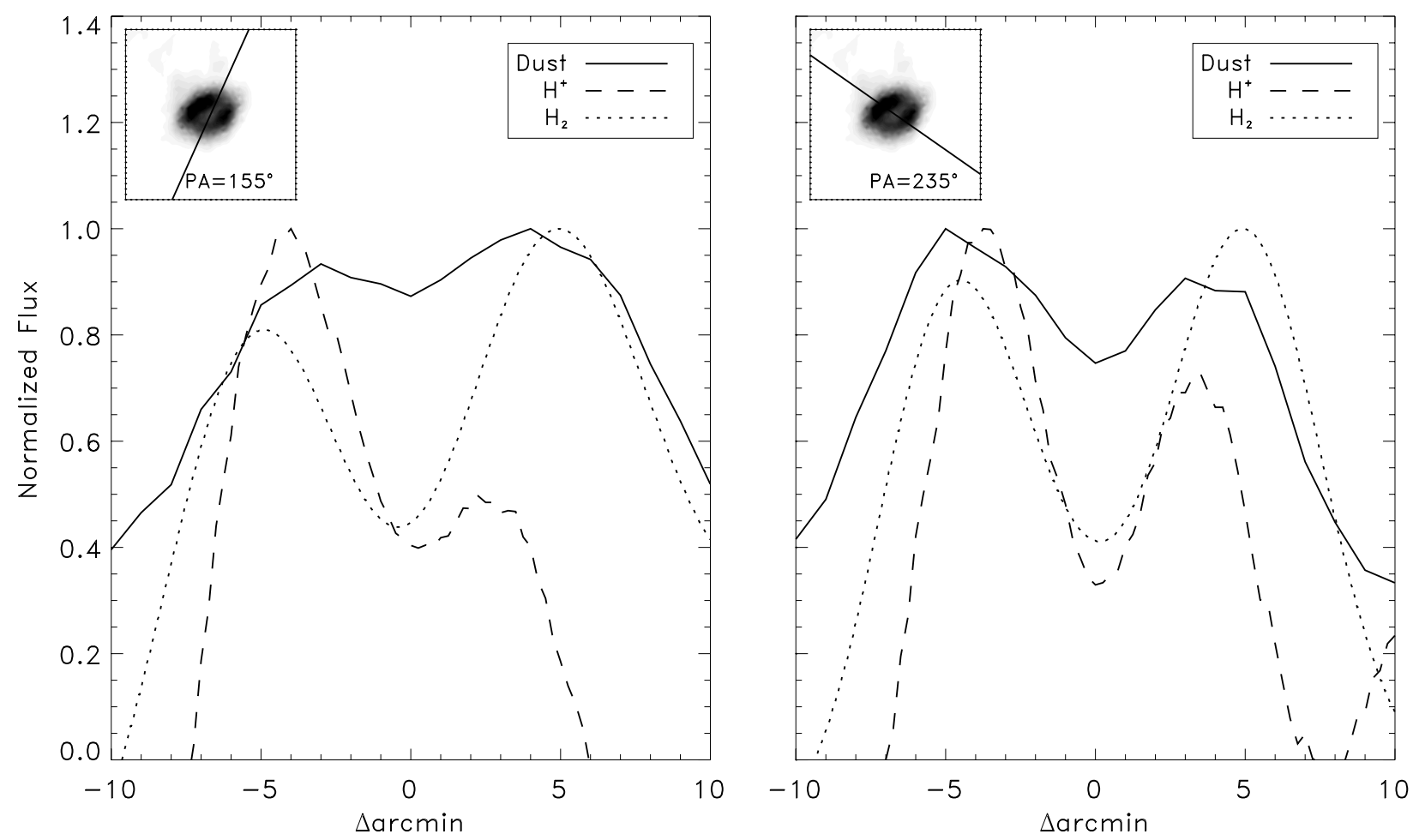

Fig. 14. Profiles of the brightness of the dust (Planck at $857 \mathrm{GHz}$ - continuous line), radio ( $\mathrm{H}+$, NVSS at $1.4 \mathrm{GHz}-$ dashed line) and $\mathrm{H}_{2}$ (dotted line) components obtained at $\mathrm{PA}=155^{\circ}(\mathrm{left})$ and $235^{\circ}$ (right). Profiles have been normalized to the Helix peak of each maps.

\section{Conclusions}

The remarkable frequency coverage of Planck has allowed us to collect important data for a small sample of Galactic PNe. These $\mathrm{PNe}$, being among the brightest of such sources, are the best studied in our Galaxy. Nevertheless, a comprehensive picture of their CSEs in both their main components, i.e., ionized gas and dust, has been provided for the first time.

In particular, the evaluation of the emission from ionized gas (free-free) allows us to constrain the thermal dust emission. The modelling of the SEDs provides us with good estimates of the physical parameters of the CSEs. From our modelling, the density, spatial distribution and total mass of ionized gas have been derived, as well as the internal radius, the extent of the dusty envelope and its mass content.

One interesting result is that, in general in the studied targets, dust and ionized gas appear to be partially co-spatial, which implies the existence of some kind of shielding mechanism to allow the dust to form, or at least to survive, in the harsh environment created by the strong UV radiation field of the central object. Such shielding can be provided by a dusty disk or a molecular torus, as pointed out by interferometric observations of young, bi-polar PNe, i.e., CRL 618, or can be in the form of clumps of material, as observed in older objects such as the Helix or NGC 6720. These shielding structures must be very common and may well be debris from material existing before the nebula was formed.

Thanks to the multi-frequency Planck measurements, the SED of the variable source CRL 618 was modelled for the first time, including both ionized gas and thermal dust contributions. This allowed us to derive important physical parameters of the CSE without variability effects.

Finally, in the case of the Helix, Planck maps enable us to perform a morphological study of the extended circumstellar material associated with the evolved PN. The dust emission was fully mapped for the first time and three main components were found, including an extended structure already seen in $\mathrm{H}_{\alpha}$ observations, probably related to a region where the slow expanding envelope interacts with the surrounding ISM. A comparison was also performed between the morphology of the dust component as traced by Planck, of the molecular gas traced by $\mathrm{H}_{2}$ near-IR observations, and of the ionized gas traced by radio $(1.4 \mathrm{GHz})$ observations. While the dust and $\mathrm{H}_{2}$ share a comparable morphology, the ionized gas appears more concentrated in the inner ring of the nebula.

Now that Planck has robustly determined the large-scale emission of this PN sample over a factor of 30 in wavelength, there is a firm basis within which to carry out detailed follow-up studies of the higher spatial and spectral-resolution properties. In particular, the results presented in this paper will provide us with a useful framework within which to plan future projects with ALMA, focusing on detailed morphological studies of both the ionized and dusty components.

Acknowledgements. The development of Planck has been supported by: ESA; CNES and CNRS/INSU-IN2P3-INP (France); ASI, CNR, and INAF (Italy); NASA and DoE (USA); STFC and UKSA (UK); CSIC, MICINN, JA and RES (Spain); Tekes, AoF and CSC (Finland); DLR and MPG (Germany); CSA (Canada); DTU Space (Denmark); SER/SSO (Switzerland); RCN (Norway); SFI (Ireland); FCT/MCTES (Portugal); and PRACE (EU). A description of the Planck Collaboration and a list of its members, including the technical or scientific activities in which they have been involved, can be found at http://www.sciops.esa.int/index.php?project=planck\& page=Planck_Collaboration The National Radio Astronomy Observatory is a facility of the National Science Foundation operated under cooperative agreement by Associated Universities, Inc. L. Cerrigone acknowledges funding from the Spanish Consejo Superior de Investigaciones Científicas through a JAEDoc research contract, co-funded by the European Social Fund. L. C. thanks the Spanish MICINN for funding support through grants AYA2009-07304 and CSD2009-00038. 
Planck Collaboration: The millimetre emission from planetary nebulae

\section{Appendix A}

Table A.1. Additional informations on the $\mathrm{mm}$ and sub-mm ancillary data used to make the SEDs.

\begin{tabular}{|c|c|c|c|c|}
\hline Facility $\ldots \ldots \ldots \ldots \ldots \ldots$ & Bands & $\begin{array}{c}\text { Beam or Aperture Size } \\
\text { [arcsec] }\end{array}$ & Notes & References \\
\hline KAO & $36 / 53 / 61 / 131[\mu \mathrm{m}]$ & $28 / 50$ & beam & Telesco \& Harper 1977 \\
\hline Hale telescope & $1[\mathrm{~mm}]$ & 55 & beam & Elias et al. 1978 \\
\hline $\mathrm{KAO} \ldots \ldots$ & $37 / 52 / 70 / 108[\mu \mathrm{m}]$ & $20 / 27 / 55$ & beam & Moseley 1980 \\
\hline NRAO Millimeter-Wave Telescope . . & $90 / 150[\mathrm{GHz}]$ & $78 / 52$ & beam & Ulich 1981 \\
\hline UKIRT $\ldots \ldots \ldots \ldots \ldots \ldots \ldots$ & $370 / 780 / 1090[\mu \mathrm{m}]$ & $40-64$ & beam & Gee et al. 1984 \\
\hline IRAM $30 \mathrm{~m}$ & $90[\mathrm{GHz}]$ & 26 & beam & Steppe et al. 1988 \\
\hline JCMT $\ldots$. & $450 / 800 / 1100 / 2000[\mu \mathrm{m}]$ & $18 / 19 / 26$ & aperture size & Hoare et al. 1992 \\
\hline JCMT $\ldots$ & $350-2000[\mu \mathrm{m}]$ & $\approx 16-27$ & beam & Knapp et al. 1993 \\
\hline IRAM $30 \mathrm{~m}$ & $250[\mathrm{GHz}]$ & $\approx 12$ & beam & Altenhoff et al. 1994 \\
\hline JCMT $\ldots .$. & $350-2000[\mu \mathrm{m}]$ & $\approx 16-27$ & beam & Sandell 1994 \\
\hline ISOPHOT $(\mathrm{mf}) \ldots$ & $7.3-200[\mu \mathrm{m}]$ & 23-184 & aperture size & Klaas et al. 2006 \\
\hline SEST $\ldots \ldots \ldots$ & $99 / 147 / 230 / 250[\mathrm{GHz}]$ & $50 / 34 / 24$ & beam & Casassus et al. 2007 \\
\hline
\end{tabular}

\section{References}

Aleman, I., Zijlstra, A. A., Matsuura, M., Gruenwald, R., \& Kimura, R. K. 2011, MNRAS, 416, 790

Aller, L. H., \& Milne, D. K. 1972, Aust. J. Phys., 25, 91

Altenhoff, W. J., Thum, C., \& Wendker, H. J. 1994, A\&A, 281, 161

Andersen, A. C., Loidl, R., \& Höfner, S. 1999, A\&A, 349, 243

Becker, R. H., White, R. L., \& Edwards, A. L. 1991, ApJS, 75, 1

Bennett, C. L., Lawrence, C. R., Burke, B. F., Hewitt, J. N., \& Mahoney, J. 1986, ApJS, 61, 1

Bernard-Salas, J., \& Tielens, A. G. G. M. 2005, A\&A, 431, 523

Bernard Salas, J., Pottasch, S. R., Beintema, D. A., \& Wesselius, P. R. 2001, A\&A, 367, 949

Bersanelli, M., Mandolesi, N., Butler, R. C., et al. 2010, A\&A, 520, A4

Buckley, D., \& Schneider, S. E. 1995, ApJ, 446, 279

Calabretta, M. R. 1982, MNRAS, 199, 141

Casassus, S., Readhead, A. C. S., Pearson, T. J., et al. 2004, ApJ, 603, 599

Casassus, S., Nyman, L.-Å., Dickinson, C., \& Pearson, T. J. 2007, MNRAS, 382, 1607

Cernicharo, J., Heras, A. M., Tielens, A. G. G. M., et al. 2001, ApJ, 546, L123

Chu, Y.-H., Gruendl, R. A., Guerrero, M. A., et al. 2009, AJ, 138, 691

Cohen, M., \& Barlow, M. J. 1980, ApJ, 238, 585

Cohen, M., \& Barlow, M. J. 2005, MNRAS, 362, 1199

Colla, G., Fanti, C., Ficarra, A., et al. 1970, A\&AS, 1, 281

Condon, J. J., \& Kaplan, D. L. 1998, ApJS, 117, 361

Condon, J. J., Cotton, W. D., Greisen, E. W., et al. 1998, AJ, 115, 1693

Cox, P., Boulanger, F., Huggins, P. J., et al. 1998, ApJ, 495, L23

Davies, J. G., Ferriday, R. J., Haslam, C. G. T., Moran, M., \& Thomasson, P. 1965, Nature, 206, 809

Davies, J. G., Faraday, R. J., Haslam, C. G. T., Moran, M., \& Thomasson, P. 1967, MNRAS, 135, 139

De Breuck, C., Tang, Y., de Bruyn, A. G., Röttgering, H., \& van Breugel, W. 2002, A\&A, 394, 59

Di Francesco, J., Johnstone, D., Kirk, H., MacKenzie, T., \& Ledwosinska, E. 2008, ApJS, 175, 277

Douglas, J. N., Bash, F. N., Bozyan, F. A., Torrence, G. W., \& Wolfe, C. 1996, AJ, 111, 1945

Draine, B. T., \& Lee, H. M. 1984, ApJ, 285, 89

Egan, M. P., Price, S. D., Kraemer, K. E., et al. 2003, VizieR Online Data Catalog: V/114

Elias, J. H., Ennis, D. J., Gezari, D. Y., et al. 1978, ApJ, 220, 25

Fazio, G. G., Hora, J. L., Allen, L. E., et al. 2004, ApJS, 154, 10

Ferland, G. J., Korista, K. T., Verner, D. A., et al. 1998, PASP, 110, 761

Fernández, R., Monteiro, H., \& Schwarz, H. E. 2004, ApJ, 603, 595

García-Segura, G., López, J. A., \& Franco, J. 2005, ApJ, 618, 919

Gathier, R., Pottasch, S. R., \& Goss, W. M. 1986, A\&A, 157, 191

Gee, G., Emerson, J. P., Ade, P. A. R., Robson, E. I., \& Nolt, I. G. 1984, MNRAS, 208,517

Gold, B., Odegard, N., Weiland, J. L., et al. 2011, ApJS, 192, 15

González-Nuevo, J., Argüeso, F., López-Caniego, M., et al. 2006, MNRAS, 369, 1603

Goodrich, R. W. 1991, ApJ, 376, 654

Gregory, P. C., \& Condon, J. J. 1991, ApJS, 75, 1011

Gregory, P. C., Scott, W. K., Douglas, K., \& Condon, J. J. 1996, ApJS, 103, 427
Griffith, M. R., Wright, A. E., Burke, B. F., \& Ekers, R. D. 1994, ApJS, 90, 179 Gruenwald, R., \& Aleman, A. 2007, A\&A, 461, 1019

Gurzadyan, G. A. 1997, The Physics and Dynamics of Planetary Nebulae (Berlin, Heidelberg, New York: Springer Verlag)

Hanner, M. 1988, Grain optical properties, Tech. rep., 22

Harris, H. C., Dahn, C. C., \& Monet, D. G. 1997, in Hipparcos - Venice '97, eds. R. M. Bonnet et al., ESA Sp. Publ., 402, 105

Healey, S. E., Romani, R. W., Taylor, G. B., et al. 2007, ApJS, 171, 61

Helou, G., \& Walker, D. W., eds. 1988, Infrared astronomical satellite (IRAS) catalogs and atlases, Volume 7: The small scale structure catalog

Higgs, L. A. 1971, MNRAS, 153, 315

Higgs, L. A. 1973, MNRAS, 161, 313

Hoare, M. G., Roche, P. F., \& Clegg, R. E. S. 1992, MNRAS, 258, 257

Hora, J. L., Latter, W. B., Allen, L. E., et al. 2004, ApJS, 154, 296

Hora, J. L., Latter, W. B., Smith, H. A., \& Marengo, M. 2006, ApJ, 652, 426

Hora, J. L., Marengo, M., Smith, H. A., Cerrigone, L., \& Latter, W. B. 2009, in The Evolving ISM in the Milky Way and Nearby Galaxies

Hughes, M. P. 1967, ApJ, 149, 377

Ivezic, Z., \& Elitzur, M. 1997, MNRAS, 287, 799

Ivezic, Z., Nenkova, M., \& Elitzur, M. 1999 [arXiv: astro-ph/9910475]

Kaftan-Kassim, M. A. 1969, ApJ, 155, 469

Khromov, G. S. 1966, Sov. Astron., 9, 705

Klaas, U., Walker, H. J., Müller, T. G., Richards, P. J., \& Schreiber, J. 2006, A\&A, 452, 523

Knapp, G. R., Sandell, G., \& Robson, E. I. 1993, ApJS, 88, 173

Kohoutek, L. 2001, A\&A, 378, 843

Kwok, S. 2008, in IAU Symp. 252, eds. L. Deng, \& K. L. Chan, 197

Kwok, S., \& Bignell, R. C. 1984, ApJ, 276, 544

Kwok, S., \& Feldman, P. A. 1981, ApJ, 247, L67

Lamarre, J., Puget, J., Ade, P. A. R., et al. 2010, A\&A, 520, A9

Large, M. I., Cram, L. E., \& Burgess, A. M. 1991, The Observatory, 111, 72

Le Marne, A. E. 1969, Aust. J. Phys., 22, 545

Leahy, J. P., Bersanelli, M., D’Arcangelo, O., et al. 2010, A\&A, 520, A8

Liu, X.-W., Barlow, M. J., Cohen, M., et al. 2001, MNRAS, 323, 343

López-Caniego, M., Herranz, D., González-Nuevo, J., et al. 2006, MNRAS, 370, 2047

Mandolesi, N., Bersanelli, M., Butler, R. C., et al. 2010, A\&A, 520, A3

Martin-Pintado, J., Bujarrabal, V., Bachiller, R., Gomez-Gonzalez, J., \& Planesas, P. 1988, A\&A, 197, L15

Mathis, J. S., Rumpl, W., \& Nordsieck, K. H. 1977, ApJ, 217, 425

Meaburn, J., Boumis, P., López, J. A., et al. 2005, MNRAS, 360, 963

Meaburn, J., Boumis, P., \& Akras, S. 2013, MNRAS, 435, 3462

Mennella, A., Butler, R. C., Curto, A., et al. 2011, A\&A, 536, A3

Menon, T. K., \& Terzian, Y. 1965, ApJ, 141, 745

Milne, D. K., \& Aller, L. H. 1975, A\&A, 38, 183

Milne, D. K., \& Aller, L. H. 1982, A\&AS, 50, 209

Miville-Deschênes, M.-A. \& Lagache, G. 2005, ApJS, 157, 302

Monteiro, H., \& Falceta-Gonçalves, D. 2011, ApJ, 738, 174

Morisset, C., \& Georgiev, L. 2009, A\&A, 507, 1517

Moseley, H. 1980, ApJ, 238, 892

Murakami, H., Baba, H., Barthel, P., et al. 2007, PASJ, 59, 369

Murphy, T., Sadler, E. M., Ekers, R. D., et al. 2010, MNRAS, 402, 2403

Nakashima, J.-i., Fong, D., Hasegawa, T., et al. 2007, AJ, 134, 2035 
O’Dell, C. R., McCullough, P. R., \& Meixner, M. 2004, AJ, 128, 2339

O'Dell, C. R., Sabbadin, F., \& Henney, W. J. 2007, AJ, 134, 1679 Ossenkopf, V., Henning, T., \& Mathis, J. S. 1992, A\&A, 261, 567 Pazderska, B. M., Gawroński, M. P., Feiler, R., et al. 2009, A\&A, 498, 463 Phillips, J. P., \& Márquez-Lugo, R. A. 2011, Rev. Mex. Astron. Astrofis., 47, 83 Phillips, J. P., Ramos-Larios, G., Schröder, K.-P., \& Contreras, J. L. V. 2009, MNRAS, 399, 1126

Phillips, J. P., Cuesta, L. C., \& Ramos-Larios, G. 2010, MNRAS, 409, 881 Planck Collaboration I. 2011, A\&A, 536, A1

Planck Collaboration II. 2011, A\&A, 536, A2

Planck Collaboration IX. 2011, A\&A, 536, A9

Planck Collaboration VIII. 2011, A\&A, 536, A8

Planck Collaboration X. 2011, A\&A, 536, A10

Planck Collaboration XI. 2011, A\&A, 536, A11

Planck Collaboration XII. 2011, A\&A, 536, A12

Planck Collaboration XIII. 2011, A\&A, 536, A13

Planck Collaboration XIV. 2011, A\&A, 536, A14

Planck Collaboration XIX. 2011, A\&A, 536, A19

Planck Collaboration XV. 2011, A\&A, 536, A15

Planck Collaboration XVI. 2011, A\&A, 536, A16

Planck Collaboration XVII. 2011, A\&A, 536, A17

Planck Collaboration XVIII. 2011, A\&A, 536, A18

Planck Collaboration XX. 2011, A\&A, 536, A20

Planck Collaboration XXI. 2011, A\&A, 536, A21

Planck Collaboration XXII. 2011, A\&A, 536, A22

Planck Collaboration XXIII. 2011, A\&A, 536, A23

Planck Collaboration XXIV. 2011, A\&A, 536, A24

Planck Collaboration XXV. 2011, A\&A, 536, A25

Planck Collaboration XXVI. 2011, A\&A, 536, A26

Planck Collaboration I. 2014, A\&A, 571, A1

Planck Collaboration II. 2014, A\&A, 571, A2

Planck Collaboration VI. 2014, A\&A, 571, A6

Planck Collaboration XXVIII. 2014, A\&A, 571, A28

Planck HFI Core Team. 2011a, A\&A, 536, A4

Planck HFI Core Team. 2011b, A\&A, 536, A6

Pottasch, S. R., \& Bernard-Salas, J. 2008, A\&A, 490, 715

Pottasch, S. R., \& Bernard-Salas, J. 2010, A\&A, 517, A95

Prinja, R. K., Hodges, S. E., Massa, D. L., Fullerton, A. W., \& Burnley, A. W. 2007, MNRAS, 382, 299

Ramos-Larios, G., Phillips, J. P., \& Cuesta, L. C. 2011, MNRAS, 411, 1245

Rengelink, R. B., Tang, Y., de Bruyn, A. G., et al. 1997, A\&AS, 124, 259

Ribes, J. C. 1969, A\&A, 3, 156

Rodríguez, L. F., Goss, W. M., \& Williams, R. 2002, ApJ, 574, 179

Rosset, C., Tristram, M., Ponthieu, N., et al. 2010, A\&A, 520, A13

Rubin, R. H. 1970, A\&A, 8, 171

Ruiz, N., Guerrero, M. A., Chu, Y.-H., \& Gruendl, R. A. 2011, AJ, 142, 91

Sabbadin, F., Turatto, M., Cappellaro, E., Benetti, S., \& Ragazzoni, R. 2004, A\&A, 416, 955

Sánchez Contreras, C. 2004, in Asymmetrical Planetary Nebulae III: Winds, Structure and the Thunderbird, eds. M. Meixner, J. H. Kastner, B. Balick, \& N. Soker, ASP Conf. Ser., 313, 89

Sánchez Contreras, C., Bujarrabal, V., Castro-Carrizo, A., Alcolea, J., \& Sargent, A. 2004, ApJ, 617, 1142

Sánchez Contreras, C., \& Sahai, R. 2004, ApJ, 602, 960

Sandell, G. 1994, MNRAS, 271, 75

Sarkar, G., \& Sahai, R. 2006, ApJ, 644, 1171

Sistla, G., Kojoian, G., \& Chaisson, E. J. 1974, ApJ, 192, 165

Slee, O. B., \& Orchiston, D. W. 1965, Aust. J. Phys., 18, 187

Speck, A. K., Meixner, M., Fong, D., et al. 2002, AJ, 123, 346

Stanghellini, L., Shaw, R. A., \& Villaver, E. 2008, ApJ, 689, 194

Steppe, H., Salter, C. J., Chini, R., et al. 1988, A\&AS, 75, 317

Surendiranath, R., \& Pottasch, S. R. 2008, A\&A, 483, 519

Szczerba, R., GÓny, S. K., Stasińska, G., Siódmiak, N., \& Tylenda, R. 2001, Ap\&SS, 275, 113

Tafoya, D., Loinard, L., Fonfría, J. P., et al. 2013, A\&A, 556, A35

Tauber, J. A., Mandolesi, N., Puget, J., et al. 2010, A\&A, 520, A1

Taylor, A. R., Pottasch, S. R., \& Zhang, C. Y. 1987, A\&A, 171, 178

Telesco, C. M., \& Harper, D. A. 1977, ApJ, 211, 475

Terzian, Y. 1966, ApJ, 144, 657

Terzian, Y. 1968, in Planetary Nebulae, eds. D. E. Osterbrock, \& C. R. O'dell, IAU Symp., 34, 87

Terzian, Y. 1969, Astrophys. Lett., 3, 87

Terzian, Y., \& Dickey, J. 1973, AJ, 78, 875

Terzian, Y., Balick, B., \& Bignell, C. 1974, ApJ, 188, 257

Thomasson, P., \& Davies, J. G. 1970, MNRAS, 150, 359

Thompson, A. R., Colvin, R. S., \& Stanley, G. J. 1967, ApJ, 148, 429

Turner, K. C., \& Terzian, Y. 1984, AJ, 89, 501

Ulich, B. L. 1981, AJ, 86, 1619
Umana, G., Leto, P., Trigilio, C., et al. 2008a, A\&A, 482, 529

Umana, G., Trigilio, C., Cerrigone, L., Buemi, C. S., \& Leto, P. 2008b, MNRAS, 386,1404

van Hoof, P. A. M. 2000, MNRAS, 314, 99

van Hoof, P. A. M., Van de Steene, G. C., Beintema, D. A., et al. 2000, ApJ, 532, 384

van Hoof, P. A. M., van de Steene, G. C., Barlow, M. J., et al. 2010, A\&A, 518, L137

Volk, K. 2003, in Planetary Nebulae: Their Evolution and Role in the Universe, eds. S. Kwok, M. Dopita, \& R. Sutherland, IAU Symp., 209, 281

Vollmer, B., Krichbaum, T. P., Angelakis, E., \& Kovalev, Y. Y. 2008, A\&A, 489, 49

Vollmer, B., Gassmann, B., Derrière, S., et al. 2010, A\&A, 511, A53

Wright, A., \& Otrupcek, R. 1990, in PKS Catalog (1990), 0

Wright, A. E., Wark, R. M., Troup, E., et al. 1991, MNRAS, 251, 330

Wright, E. L., Chen, X., Odegard, N., et al. 2009, ApJS, 180, 283

Wright, E. L., Eisenhardt, P. R. M., Mainzer, A. K., et al. 2010, AJ, 140, 1868

Wyrowski, F., Schilke, P., Thorwirth, S., Menten, K. M., \& Winnewisser, G. 2003, ApJ, 586, 344

Zacchei, A., Maino, D., Baccigalupi, C., et al. 2011, A\&A, 536, A5

Zhang, C. Y., \& Kwok, S. 1991, A\&A, 250, 179

Zhang, Y., Hsia, C.-H., \& Kwok, S. 2012, ApJ, 755, 53

Zijlstra, A. A., van Hoof, P. A. M., \& Perley, R. A. 2008, ApJ, 681, 1296

1 APC, AstroParticule et Cosmologie, Université Paris Diderot, CNRS/IN2P3, CEA/lrfu, Observatoire de Paris, Sorbonne Paris Cité, 10 rue Alice Domon et Léonie Duquet, 75205 Paris Cedex 13, France

2 Aalto University Metsähovi Radio Observatory and Dept of Radio Science and Engineering, PO Box 13000, 00076 Aalto, Finland

3 African Institute for Mathematical Sciences, 6-8 Melrose Road, 7945 Muizenberg, Cape Town, South Africa

4 Agenzia Spaziale Italiana Science Data Center, via del Politecnico snc, 00133 Roma, Italy

5 Agenzia Spaziale Italiana, Viale Liegi 26, 00198 Roma, Italy

6 Astrophysics Group, Cavendish Laboratory, University of Cambridge, J J Thomson Avenue, Cambridge CB3 0HE, UK

7 Astrophysics \& Cosmology Research Unit, School of Mathematics, Statistics \& Computer Science, University of KwaZulu-Natal, Westville Campus, Private Bag X54001, 4000 Durban, South Africa

8 CITA, University of Toronto, 60 St. George St., Toronto, ON M5S 3H8, Canada

9 CNRS, IRAP, 9 Av. colonel Roche, BP 44346, 31028 Toulouse Cedex 4, France

10 California Institute of Technology, Pasadena, California, USA

11 Centro de Astrobiología (INTA-CSIC), 28850 Torrejón de Ardoz, Madrid, Spain

12 Computational Cosmology Center, Lawrence Berkeley National Laboratory, Berkeley, California, USA

13 DSM/Irfu/SPP, CEA-Saclay, 91191 Gif-sur-Yvette Cedex, France

14 DTU Space, National Space Institute, Technical University of Denmark, Elektrovej 327, 2800 Kgs. Lyngby, Denmark

15 Département de Physique Théorique, Université de Genève, 24, Quai E. Ansermet,1211 Genève 4, Switzerland

16 Departamento de Física Fundamental, Facultad de Ciencias, Universidad de Salamanca, 37008 Salamanca, Spain

17 Departamento de Física, Universidad de Oviedo, Avda. Calvo Sotelo s/n, 33007 Oviedo, Spain

18 Department of Astrophysics/IMAPP, Radboud University Nijmegen, PO Box 9010, 6500 GL Nijmegen, The Netherlands

19 Department of Physics \& Astronomy, University of British Columbia, 6224 Agricultural Road, Vancouver, British Columbia, Canada

20 Department of Physics and Astronomy, Dana and David Dornsife College of Letter, Arts and Sciences, University of Southern California, Los Angeles, CA 90089, USA

21 Department of Physics and Astronomy, University College London, London WC1E 6BT, UK 
22 Department of Physics, Florida State University, Keen Physics Building, 77 Chieftan Way, Tallahassee, Florida, USA

23 Department of Physics, Gustaf Hällströmin katu 2a, University of Helsinki, 00014 Helsinki, Finland

24 Department of Physics, Princeton University, Princeton, New Jersey, USA

25 Department of Physics, University of California, Santa Barbara, California, USA

26 Department of Physics, University of Illinois at Urbana-Champaign, 1110 West Green Street, Urbana, Illinois, USA

27 Dipartimento di Fisica e Astronomia G. Galilei, Università degli Studi di Padova, via Marzolo 8, 35131 Padova, Italy

28 Dipartimento di Fisica e Scienze della Terra, Università di Ferrara, via Saragat 1, 44122 Ferrara, Italy

29 Dipartimento di Fisica, Università La Sapienza, P. le A. Moro 2, 00185 Roma, Italy

30 Dipartimento di Fisica, Università degli Studi di Milano, via Celoria 16, 20133 Milano, Italy

31 Dipartimento di Fisica, Università degli Studi di Trieste, via A. Valerio 2, 34127 Trieste, Italy

32 Dipartimento di Fisica, Università di Roma Tor Vergata, via della Ricerca Scientifica 1, 00133 Roma, Italy

33 Discovery Center, Niels Bohr Institute, Blegdamsvej 17, 2100 Copenhagen, Denmark

34 Dpto. Astrofísica, Universidad de La Laguna (ULL), 38206 La Laguna, Tenerife, Spain

35 European Space Agency, ESAC, Planck Science Office, Camino bajo del Castillo, s/n, Urbanización Villafranca del Castillo, 28691 Villanueva de la Cañada, Madrid, Spain

36 European Space Agency, ESTEC, Keplerlaan 1, 2201 AZ Noordwijk, The Netherlands

37 Facoltà di Ingegneria, Università degli Studi e-Campus, via Isimbardi 10, 22060 Novedrate (CO), Italy

38 Harvard-Smithsonian Center for Astrophysics, 60 Garden Street, Cambridge, MA 02138, USA

39 Helsinki Institute of Physics, Gustaf Hällströmin katu 2, University of Helsinki, 00014 Helsinki, Finland

40 INAF - Osservatorio Astrofisico di Catania, via S. Sofia 78, 95123 Catania, Italy

41 INAF - Osservatorio Astronomico di Padova, Vicolo dell'Osservatorio 5, 35122 Padova, Italy

42 INAF - Osservatorio Astronomico di Roma, via di Frascati 33, 00040 Monte Porzio Catone, Italy

43 INAF - Osservatorio Astronomico di Trieste, via G.B. Tiepolo 11, 34143 Trieste, Italy

44 INAF Istituto di Radioastronomia, via P. Gobetti 101, 40129 Bologna, Italy

45 INAF/IASF Bologna, via Gobetti 101, 40129 Bologna, Italy

46 INAF/IASF Milano, via E. Bassini 15, 20133 Milano, Italy

47 INFN, Sezione di Bologna, via Irnerio 46, 40126, Bologna, Italy

48 INFN, Sezione di Roma 1, Università di Roma Sapienza, Piazzale Aldo Moro 2, 00185 Roma, Italy

49 INFN/National Institute for Nuclear Physics, via Valerio 2, 34127 Trieste, Italy

50 Imperial College London, Astrophysics group, Blackett Laboratory, Prince Consort Road, London, SW7 2AZ, UK

51 Infrared Processing and Analysis Center, California Institute of Technology, Pasadena, CA 91125, USA

52 Institut d'Astrophysique Spatiale, CNRS (UMR 8617) Université Paris-Sud 11, Bâtiment 121, 91405 Orsay, France

53 Institut d'Astrophysique de Paris, CNRS (UMR 7095), 98bis Boulevard Arago, 75014 Paris, France
${ }^{54}$ Institute for Space Sciences, 077125 Bucharest-Magurale, Romania

55 Institute of Astronomy, University of Cambridge, Madingley Road, Cambridge CB3 0HA, UK

56 Institute of Theoretical Astrophysics, University of Oslo, Blindern, 0315 Oslo, Norway

57 Instituto de Astrofísica de Canarias, C/Vía Láctea s/n, 38205 La Laguna, Tenerife, Spain

58 Instituto de Física de Cantabria (CSIC-Universidad de Cantabria), Avda. de los Castros s/n, Santander, Spain

59 Jet Propulsion Laboratory, California Institute of Technology, 4800 Oak Grove Drive, Pasadena, California, USA

60 Jodrell Bank Centre for Astrophysics, Alan Turing Building, School of Physics and Astronomy, The University of Manchester, Oxford Road, Manchester, M13 9PL, UK

61 Kavli Institute for Cosmology Cambridge, Madingley Road, Cambridge, CB3 OHA, UK

${ }^{62}$ LAL, Université Paris-Sud, CNRS/IN2P3, 91898 Orsay, France

63 LERMA, CNRS, Observatoire de Paris, 61 Avenue de l'Observatoire, 75014 Paris, France

${ }^{64}$ Laboratoire AIM, IRFU/Service d'Astrophysique - CEA/DSM CNRS - Université Paris Diderot, Bât. 709, CEA-Saclay, 91191 Gif-sur-Yvette Cedex, France

65 Laboratoire Traitement et Communication de l'Information, CNRS (UMR 5141) and Télécom ParisTech, 46 rue Barrault, 75634 Paris Cedex 13, France

66 Laboratoire de Physique Subatomique et de Cosmologie, Université Joseph Fourier Grenoble I, CNRS/IN2P3, Institut National Polytechnique de Grenoble, 53 rue des Martyrs, 38026 Grenoble Cedex, France

${ }^{67}$ Laboratoire de Physique Théorique, Université Paris-Sud 11 \& CNRS, Bâtiment 210, 91405 Orsay, France

${ }^{68}$ Lawrence Berkeley National Laboratory, Berkeley, California, USA

69 Max-Planck-Institut für Astrophysik, Karl-Schwarzschild-Str. 1, 85741 Garching, Germany

70 National University of Ireland, Department of Experimental Physics, Maynooth, Co. Kildare, Ireland

71 Niels Bohr Institute, Blegdamsvej 17, 2100 Copenhagen, Denmark

72 Observational Cosmology, Mail Stop 367-17, California Institute of Technology, Pasadena, CA, 91125, USA

73 Optical Science Laboratory, University College London, Gower Street, London, UK

74 SISSA, Astrophysics Sector, via Bonomea 265, 34136 Trieste, Italy

75 School of Physics and Astronomy, Cardiff University, Queens Buildings, The Parade, Cardiff, CF24 3AA, UK

76 Space Sciences Laboratory, University of California, Berkeley, California, USA

77 Special Astrophysical Observatory, Russian Academy of Sciences, Nizhnij Arkhyz, Zelenchukskiy region, 369167 Karachai-Cherkessian Republic, Russia

78 UPMC Univ. Paris 06, UMR 7095, 98bis Boulevard Arago, 75014 Paris, France

79 Universidad de Chile, Casilla 36-D Santiago, Chile

80 Université de Toulouse, UPS-OMP, IRAP, 31028 Toulouse Cedex 4, France

81 University of Granada, Departamento de Física Teórica y del Cosmos, Facultad de Ciencias, 18071 Granada, Spain

82 University of Granada, Instituto Carlos I de Física Teórica y Computacional, Granada, Spain

83 Warsaw University Observatory, Aleje Ujazdowskie 4, 00-478 Warszawa, Poland 